\title{
A combined experimental and numerical study of laminar and turbulent non-piloted oxy-fuel jet flames using a direct comparison of the Rayleigh signal
}

\author{
Franziska Hunger • Meor F. Zulkifli • \\ Benjamin A. O. Williams • Frank Beyrau • \\ Christian Hasse
}

Received: date / Accepted: date

\begin{abstract}
In the present study laminar and turbulent oxy-fuel jet flames are investigated both experimentally and numerically with emphasis on the direct comparison of the Rayleigh signal. The Rayleigh signal was measured for both flame setups, correcting for background light appropriately. Two downstream regions were recorded for the laminar flame and three for the turbulent flame. Equivalently, the signal was processed numerically based on the numerical species data and temperature.

The laminar flame was used for validating the procedure of processing the Rayleigh signal. Both the numerical species data and the temperature are known from detailed simulations, so a predicted Rayleigh signal can easily be obtained. Further, the influence of the choice of the kinetic mechanism, radiation and diffusion model was investigated. In contrast, in the turbulent Large Eddy Simulation, the Rayleigh signal has to be computed using an appropriate turbulence-chemistry interaction model in order to obtain the Reynolds-filtered Rayleigh signal which is of non-linear nature. In the present investigation, the Rayleigh signal was incorporated in the flamelet/progress variable approach. The statistics of the experimental and numerical Rayleigh signal were then compared.

The proposed procedure of directly comparing the experimental and predicted Rayleigh signal was shown to be advantageous in model validation especially in turbulent flame configurations. The procedure enables accurate model validation across an entire $2 \mathrm{D}$ field of view whilst using a realistic fuel-oxidizer combination and reducing experimental complexity.
\end{abstract}

F. Hunger · C. Hasse

Chair of Numerical Thermo-Fluid Dynamics

ZIK Virtuhcon

TU Bergakademie Freiberg

Freiberg, Germany

Tel.: +49-3731-394821

Fax: +49-3731-394555

E-mail: franziska.hunger@vtc.tu-freiberg.de

Meor F. Zulkifli · Benjamin A. O. Williams · Frank Beyrau

Department of Mechanical Engineering

Imperial College London

London, Great Britain 
Keywords Rayleigh scattering $\cdot$ LES $\cdot$ DNS flamelet-progress variable $\cdot$ model validation

\section{Introduction}

In most combustion processes air is used as the oxidizer. However, oxy-fuel combustion processes offer advantages such as increased thermal efficiency, higher processing rates, reduced flue gas volumes and reduced pollutant emissions at higher oxygen concentrations [4, 47]. Oxy-fuel flames are prone to differential diffusion effects [22, 49], complex radiation [11, 29, 51, higher flame temperatures [27, 52] and an alteration of the chemical path when $\mathrm{CO}_{2}$ is added 38 . Further, the experimental investigation of such systems can become more complicated. Soot formation [56, significantly altered chemical paths [18, 30, 38, and high heat losses [46] due to substituting $\mathrm{N}_{2}$ for $\mathrm{CO}_{2}$ cause several challenges for experimentalists.

Following an increasing number of investigations of complex fuels or fuel/oxidizer mixtures, significant advances have been made to improve the interplay between experiments and numerics in order to better understand the underlying physical and thermochemical phenomena, e.g. 2, 7]. However, experimental measurements are usually postprocessed in order to obtain desired physical quantities (e.g. species mass/mole fractions or temperature) to enable comparison to equivalent numerical results. Using this approach, potential inaccuracies and assumptions of data processing are transferred to the experimental/numerical comparison.

Considering Rayleigh measurements particularly, the signal in oxy-fuel flames is highly affected by a variation of effective Rayleigh cross section throughout the flame compared to air flames as demonstrated in [5, 10. This makes evaluation of the temperature based on Rayleigh measurements prone to inaccuracies, as discussed later.

In the specific case of an $\mathrm{H}_{2}$-air flame, Stepowski and Cabot [53] demonstrated the use of a strained flame library in order to convert the Rayleigh signal into temperature and mixture fraction data. To apply this method to a more complex oxy-fuel flame, many assumptions are required which would introduce significant uncertainty. Further, Fuest et al. [16] performed combined Raman/Rayleigh measurements in DME flames. Even when measuring the species composition via the Raman technique, evaluating the temperature based on the Rayleigh signal can become complicated since the Raman signal can only obtain a mixture of different hydrocarbons in one channel. Hence, Fuest et al. [16] needed to apply a model for evaluating the species cross section of this hydrocarbon mixture. In order to overcome the problems of converting measurements to physical quantities, the comparison of the actual experimental signal and the signal predicted by numerical simulations was suggested by Connelly et al. 8 . The advantages of this procedure were named to be simpler experiments requiring fewer measurements, and a better signal-to-noise ratio which leads to reduced uncertainties. For turbulent flames, this approach is also important because simultaneous measurements of a sufficient number of quantities may not be possible. Connelly et al. 8] applied the procedure to NO-LIF and luminosity measured from laminar sooting and non-sooting flames. A similar approach has been applied to a turbulent DME flame in order to investigate the $\mathrm{CH}_{2} \mathrm{O}$ - and the OH-LIF signal [9], where the turbulent simulation was performed with an LES-CMC approach. Further, a first investigation and validation of the incorporation of different experimental signals into the flamelet/progress variable (FPV) model is given in 43] for the same DME flame. A first attempt to compare experimental and 
numerical Rayleigh signals was presented by Schießl et al. [48, who used steady and unsteady laminar counterflow flame computations to construct a two-dimensional manifold with the mixture fraction and a reduced temperature as independent variables. The latter can be regarded as a normalized progress variable, which is quite common in FPV modeling strategies. Schießl et al. 48] then computed the polarized and depolarized Rayleigh signal based on the flamelet temperature and species information. By using both experimental Rayleigh signals, the authors determined the species and the temperature by a Newton iteration and showed their predicted spatial distribution for a single realization. Although no quantitative comparison was possible, using additional CO- and OH-LIF data recorded simultaneously with Rayleigh, a good qualitative agreement between the results from the two-dimensional flamelet manifold and the experiment was observed.

In this report, the idea is extended by looking at a laminar and a turbulent oxy-fuel flame. Rayleigh scattering was the technique chosen to generate data since it provides data for validation across the field of view. Alternatives are generally spatially restricted, such as OH-PLIF to the flame zone or simultaneous Raman/Rayleigh limited to 1D. The Rayleigh technique also offers a quantitative measurement, compared to OH-PLIF which is usually qualitative (please refer to appendix A for further discussion of OH-PLIF in this application). As discussed above, previous results [48, showed that using numerical Rayleigh signals based on precomputed flamelet solutions might be suitable for direct comparison with experimental Rayleigh signals. We directly include the Rayleigh signal both in the laminar and the turbulent multi-dimensional simulations, which we consider fully-coupled as compared to previous investigations which used a post-processing approach only. In the laminar calculation, all chemical and transport processes are fully resolved. This allows to directly evaluate modeling assumptions and their effect on the Rayleigh signal. By direct comparison to the single experimental Rayleigh signal, the influence of the radiation model, the chemical mechanism and the diffusion modeling approach can be quantified. For the turbulent case, the Rayleigh signal is computed based on the laminar flamelet calculation and tabulated along with other physical quantities such as the species and the temperature for a Large Eddy Simulation (LES) using the flamelet/progress variable approach. This tabulation also includes a model for the subgrid fluctuations, since not all processes can be resolved in an LES. Here we specifically look at the turbulent statistics such as the mean and the variance of the Rayleigh signal and assess their comparability. For selected positions in the flame, we additionally look in detail at the full experimental and numerical distribution function. While the work of Schießl et al. 48, already suggested the applicability of the procedure looking at single snapshots, the focus here is on the turbulent statistics, which is a common measure for LES of turbulent jet flames. Here, the advantage of the proposed procedure is that the statistics can easily be evaluated in the entire measured 2D domain. This is discussed below in detail.

The remainder of this paper is structured as follows. Section 2 discusses the experimental approach, describing the burner in Sec. 2.1. analyzing the Rayleigh scattering properties of fuel and oxidizer in Sec. 2.2 and discussing Rayleigh signal processing in Sec. 2.3. The mathematical models are described in Sec. 3, for the laminar flame in Sec. 3.1 and for the turbulent flame in Sec. 3.2 The numerical procedure for processing the Rayleigh signal is given in Sec. 3.3 Finally, the results of this oxy-fuel flame investigation are presented in Sec. 4 and a conclusion is given in Sec. 5 


\section{Experimental Approach}

In the following, the experimental approach is outlined. First, the burner is briefly described in Sec. 2.1. Further, the fuel and oxidizer composition is discussed in Sec. 2.2 where details of the variation in the Rayleigh cross section are given and a comparison to common methane-air combustion is made. Finally, the procedure of Rayleigh signal processing is explained in Sec. 2.3. For illustration, the figure in appendix B shows a color photograph of the oxy-fuel flame.

\subsection{Burner and Flame Details}

The burner, see Fig. 3 consists of a $5 \mathrm{~mm}$ inner diameter nozzle with $0.5 \mathrm{~mm}$ wall thickness surrounded by two co-flows with inner diameters of $95.5 \mathrm{~mm}$ and $211.56 \mathrm{~mm}$, respectively. The nozzle protrudes $40 \mathrm{~mm}$ above the co-flow exit plane, and has a supply tube 100 diameters in length in order to ensure a fully developed flow when it reaches the nozzle tip. The co-flow gases pass through a flow-homogenizing layer of glass beads sandwiched between perforated plates before entering a $100 \mathrm{~mm}$ tall section of honeycomb used to straighten the flow. All gases were supplied from cylinders and controlled using mass flow controllers (Bronkhorst El-Flow series) through a custom LABVIEW program. The burner was translated in the axial direction to change the flame measurement region.

Two flames were investigated, one laminar and one turbulent. Fuel was supplied to the nozzle, oxidizer to the inner co-flow, and air to the outer co-flow at a matching velocity to the inner co-flow in order to prevent entrainment of room air.

\subsection{Fuel and Oxidizer Selection}

The compositions of the fuel and oxidizer were carefully chosen to create a stable, representative oxy-fuel flame suitable for investigation with the Rayleigh scattering technique. In the oxy-fuel flame, the goal was to minimize soot formation and generate a flame temperature far in excess of air-fed combustion. An attempt was also made to maintain a constant scattering cross section during the reaction, however, the optimal composition has still appreciable variations that cannot be neglected. The details of fuel and oxidizer selection will now be discussed.

In oxy-fuel combustion, $\mathrm{CO}_{2}$ is used as a diluent rather than $\mathrm{N}_{2}$ which can have a cooling effect, primarily due to the higher heat capacity of $\mathrm{CO}_{2}$ compared to $\mathrm{N}_{2}$. Samaniego and Mantel [46] also observed that the radiative heat loss of a $\mathrm{CO}_{2}$ diluted flame can be up to four times higher than an equivalent $\mathrm{N}_{2}$ diluted flame (although still a small, $<1 \%$, contribution in absolute terms). Further, Ditaranto and Hals [1] reported that at least $30 \% \mathrm{O}_{2}$ is required to achieve air-like combustion stability. A numerical calculation was performed in CHEMKIN-PRO [25] to determine the oxygen concentration needed in this experiment, and $68 \%$ (in the oxidizer stream by volume) is required to achieve the temperature of interest $(>2400 \mathrm{~K})$. Oxidizer composition also plays an important role in soot formation. Wang et al. [56] investigated propane flames burning in oxidizers of varying oxygen content. The authors found that the measured soot volume fraction increases to a peak between $30 \%-40 \%$ oxygen content in the oxidizer, and decreases as further oxygen is added owing to promotion of soot oxidation. 


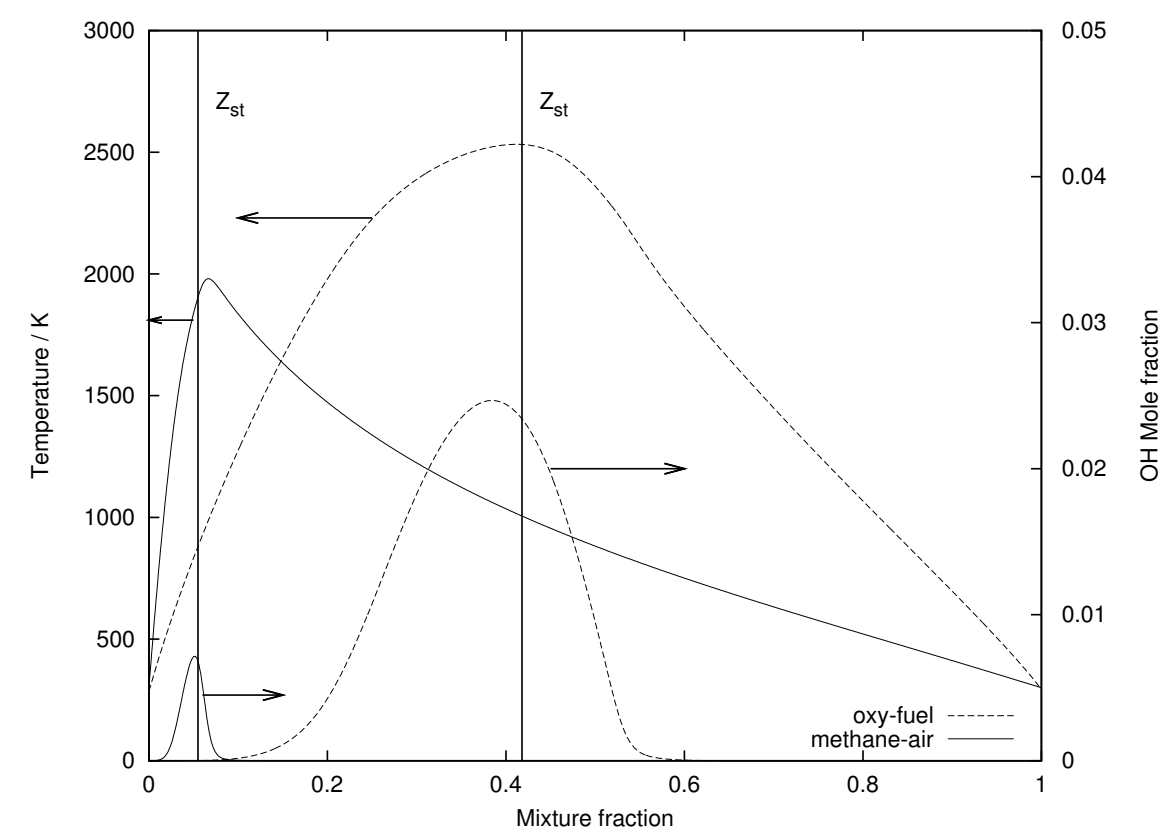

Fig. 1: Temperature and $\mathrm{OH}$ mole fraction of a methane-air flame and oxy-fuel flame obtained from a laminar flamelet solution with $\chi_{s t}=10 \mathrm{~s}^{-1}$. In addition, the stoichiometric mixture fraction $Z_{s t}$ is marked.

Localized flame extinction is likely to happen when the turbulent mixing rate is higher than the chemical reaction rate, leading to lift-off or blow-off. $\mathrm{As} \mathrm{CH}_{4}$ has a relatively low chemical reaction rate, the addition of $\mathrm{H}_{2}$ in the fuel mixture acts to increase its global reaction rate. For example, 49] reported that localized extinction occurred as a result of either decreasing the amount of $\mathrm{H}_{2}$ in the fuel mixture or increasing the Reynolds number of the jet.

The Rayleigh signal could be directly converted into temperature by ensuring a constant Rayleigh cross section throughout the combustion process (from reactants to products) as demonstrated by [5, 10] whose mixtures varied in cross section by less than $3 \%$. However, in high temperature oxy-fuel combustion there is in general significant production of radicals $(\mathrm{H}, \mathrm{O}, \mathrm{OH})[30$. Figure 1 shows the results of laminar flamelet calculations comparing our oxy-fuel system to methane-air combustion, demonstrating significantly increased $\mathrm{OH}$ production (and temperature) in the oxy-fuel case. The presence of $\mathrm{CO}_{2}$ in the present work also causes significant proportions of $\mathrm{CO}$ to be generated following $\mathrm{CO}_{2}$ dissociation. Despite its negative effect on the consistency of the Rayleigh cross section during combustion, the addition of $\mathrm{CO}_{2}$ can be advantageous. Du et al. 12 found that $\mathrm{CO}_{2}$ helps reduce soot formation and suggested this is due to the decreasing concentration of $\mathrm{H}$ radicals which are consumed in the reaction $\mathrm{CO}_{2}+\mathrm{H} \rightleftharpoons \mathrm{CO}+\mathrm{OH} . \mathrm{CO}_{2}$ dilution at high temperature $(2000 \mathrm{~K}-3000 \mathrm{~K})$ was also agreed by Mazas et al. 31] to lead to a significant decrease in soot formation.

Figure 2 shows the predicted variation of temperature, species mole fraction, Rayleigh ratio (experimental and numerical evaluation see Secs. 2.3 and 3.3 and Rayleigh cross 

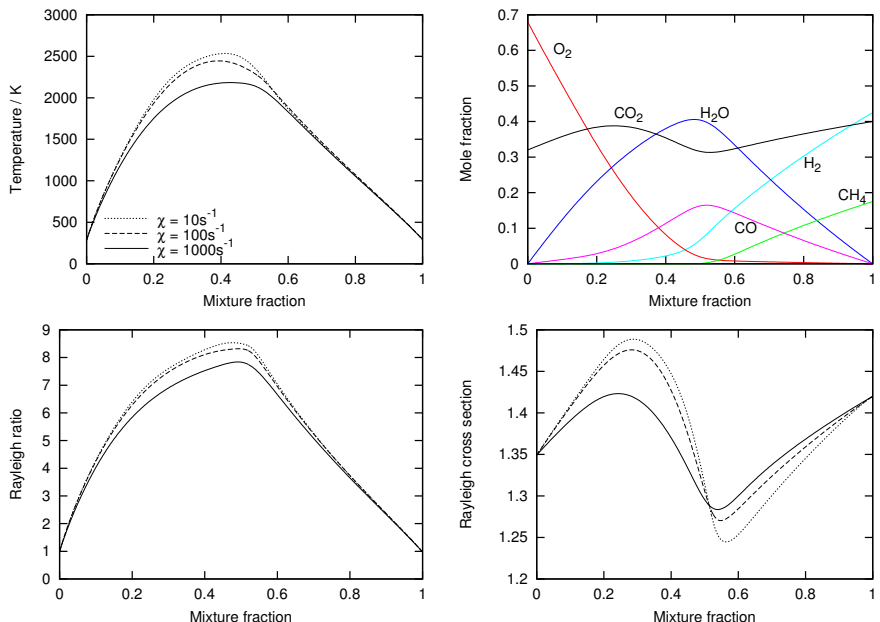

Fig. 2: Temperature, mole fraction, Rayleigh ratio and Rayleigh cross section from the laminar flamelet solution for the investigated fuel $\left(17.5 \% \mathrm{CH}_{4}, 40 \% \mathrm{CO}_{2}, 42.5 \% \mathrm{H}_{2}\right.$ by volume) and oxidizer $\left(68 \% \mathrm{O}_{2}, 32 \% \mathrm{CO}_{2}\right.$ by volume); species profiles shown for $\chi_{s t}=1000 s^{-1}$.

section as a function of mixture fraction as calculated in a unity Lewis-number laminar flamelet calculation using GRI-MECH 3.0 for our chosen mixtures - fuel: $17.5 \% \mathrm{CH}_{4}$, $40 \% \mathrm{CO}_{2}, 42.5 \% \mathrm{H}_{2}$ by volume and oxidizer: $68 \% \mathrm{O}_{2}, 32 \% \mathrm{CO}_{2}$ by volume for a range of expected strain rates. The species concentrations, indicating peak production of $\mathrm{CO}$ and $\mathrm{H}_{2} \mathrm{O}$ at a stoichiometric mixture fraction value of 0.418 are shown for an expected scalar dissipation rate of $\chi_{s t}=1000 s^{-1}$. The predicted variation in Rayleigh cross section (bottom right) was calculated using species-specific cross section values from [16], and shows that the aforementioned production of $\mathrm{CO}$ and $\mathrm{H}_{2} \mathrm{O}$ (top right) has a noticeable impact on the overall cross section owing to their smaller cross sections compared to $\mathrm{CO}_{2}$. Note that $\mathrm{CO}_{2}$ was mixed into the fuel stream to moderate this variation, and hence produces a larger cross section of the fuel compared to the oxidizer. The relative fluctuation in cross section is between 5 and $15 \%$. The top left subplot in Fig. 2 shows the relationship between temperature and strain rate. Since strain rate reduces further downstream, a higher temperature is to be expected. Finally, the bottom left subplot indicates that the Rayleigh ratio increases as strain rate reduces. For completeness, an analysis of differential diffusion effects is included in appendix E but not discussed at length here, owing to the dominance of turbulent mixing in this system. The analysis shows that the assumption of unity Lewis number gives best agreement with the experimental data.

For "traditional" validation, experimental measurements are converted to temperature data by assuming constant Rayleigh scattering cross section during the reaction. However, when a variation in the Rayleigh cross section is present, this conversion becomes prone to inaccuracies. In our case, the requirements for the fuel-oxidizer composition (e.g. flame temperature, soot formation) while maintaining a constant Rayleigh cross section, still lead to a variation of more than $5 \%$. Thus, the direct comparison between the experimentally-measured ratio of Rayleigh signals and a numerically-predicted ra- 
Table 1: Experimental Conditions

\begin{tabular}{|l|l|l|}
\hline Flow Conditions & Turbulent & Laminar \\
\hline Jet flow rate & $\begin{array}{l}8.6 \cdot 10^{-4} \mathrm{~kg} / \mathrm{s} \\
(\mathrm{Re}=18,000)\end{array}$ & $\begin{array}{l}4.8 \cdot 10^{-5} \mathrm{~kg} / \mathrm{s} \\
(\mathrm{Re}=850)\end{array}$ \\
\hline Coflow rate & $\begin{array}{l}1.1 \cdot 10^{-2} \mathrm{~kg} / \mathrm{s} \\
\left(\mathrm{U}_{\mathrm{bulk}}=1 \mathrm{~m} / \mathrm{s}\right)\end{array}$ & $\begin{array}{l}3.2 \cdot 10^{-3} \mathrm{~kg} / \mathrm{s} \\
\left(\mathrm{U}_{\mathrm{bulk}}=0.3 \mathrm{~m} / \mathrm{s}\right)\end{array}$ \\
\hline Ambient Temperature & $293 \mathrm{~K}$ & $293 \mathrm{~K}$ \\
\hline Fuel Temperature & $292 \mathrm{~K}$ & $292 \mathrm{~K}$ \\
\hline Oxidizer Temperature & $285 \mathrm{~K}$ & $285 \mathrm{~K}$ \\
\hline $\begin{array}{l}\text { Measurement locations } \\
\text { (downstream from nozzle) }\end{array}$ & $23-48 \mathrm{~mm}, 48-73 \mathrm{~mm}, 98-$ & $23-48 \mathrm{~mm}, 60-85 \mathrm{~mm}$ \\
\cline { 1 - 2 } & $123 \mathrm{~mm}$, shown in Fig. 15 & \multicolumn{2}{|l}{} \\
\cline { 1 - 2 } & \multicolumn{2}{|l}{}
\end{tabular}

tio of Rayleigh signals reduces the uncertainties of the conversion to a Rayleigh-inferred temperature and it further relieves setting up the experiments by not restricting the fuel-oxidizer composition to obtain constant Rayleigh cross sections. Thus, a procedure to obtain the equivalent Rayleigh signal from numerical simulations was developed and this is described in Sec. 3.3 .

\subsection{Rayleigh Scattering}

\subsubsection{Experimental Setup}

The Rayleigh scattering measurements were performed using the experimental setup illustrated in Fig. 3 Four gas cylinders were used that contain helium for stray light correction, air for the shielding co-flow, the oxidizer mixture and the fuel mixture, respectively. The outputs of four frequency-doubled Nd:YAG laser heads (THALES Multi-channel system) at $532 \mathrm{~nm}$ were spatially overlapped, producing a combined energy of around $1.8 \mathrm{~J} /$ shot. The laser system was operated in burst mode, producing a batch of four sequential $10 \mathrm{~ns}$ duration pulses every $100 \mathrm{~ms}$, and thereby limiting the peak intensity through the harmonic crystals. The polarization of the laser output was rotated to vertical and the beam was shaped using lenses into a laser sheet with a thickness of approximately $120 \mu \mathrm{m}$ at the center of the imaged region and a height of $30 \mathrm{~mm}$. An iris was positioned in the beam path near to the burner to block any stray light above and below the main laser sheet. The Rayleigh scattered light from the measurement volume was imaged onto an unintensified interline-transfer CCD camera (LaVision Imager Intense, set to $1 \mu$ s exposure duration) using a camera lens (f/1.2, $\mathrm{f}=50 \mathrm{~mm})$ and isolated with an interference filter $\left(\lambda_{c}=532 \mathrm{~nm}, \Delta \lambda_{\mathrm{FWHM}}=3 \mathrm{~nm}\right)$. After $2 \times 2$ binning this yielded an imaging region of $33 \mathrm{~mm} \times 25 \mathrm{~mm}$ with an object resolution of $50 \mu \mathrm{m} /$ pixel. Due to a large flow rate of oxidizer in the co-flow, the effect of Joule-Thompson cooling was apparent as the gas mixture was forced through valves from the gas cylinder to the burner, leading to a temperature drop. Previous studies have adopted an in-line heater to attempt to maintain a constant gas temperature 49. However, we chose to measure the gas temperature using a thermocouple both before and after each data run. These values were then used as inputs to the numerical analysis. Details of the flow conditions are given in Tab. 1 . 


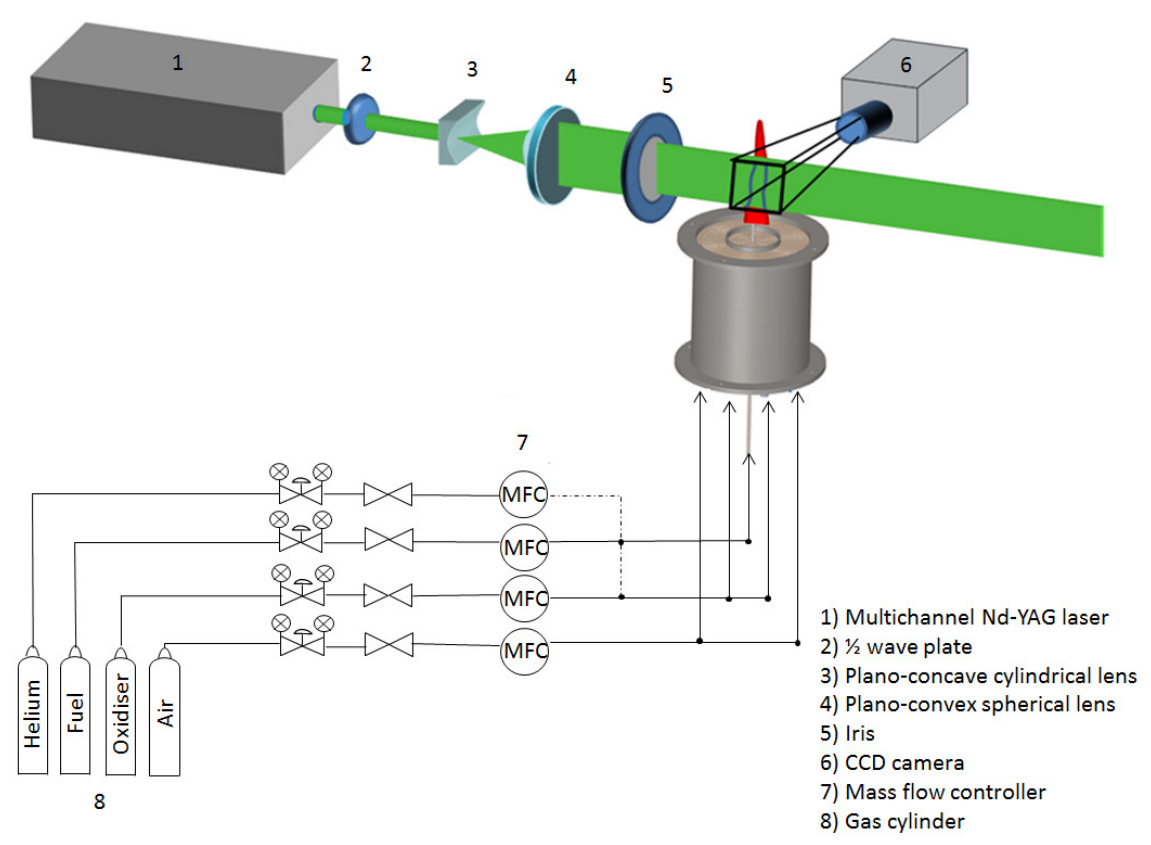

Fig. 3: Rayleigh scattering experimental setup.

\subsubsection{Signal Processing}

$\llcorner$ Five experimental conditions were investigated: three measurement locations for the turbulent flame and two for the laminar flame, as given in Table 1. For each experimental configuration, three sets of data were collected, each one containing 500-1000 images. The first image set flooded the imaging region with helium, the second swapped the helium for oxidizer and the third employed the appropriate flame settings. This volume of data was chosen to enable reliable statistical analysis. The raw experimental data was processed before comparison with the numerical predictions. This processing was intended to retrieve the true Rayleigh signal by removing contributions from stray light (i.e. contributions from room lights and direct laser scatter) and camera dark current. Camera dark current is accounted for by collecting a set of images with the lens cap left on and forming the average. Stray light can be calculated by taking two sets of reference images, with different gases flooding the measurement region. In these tests, we used the helium and the oxidizer image sets. The raw pixel signal, $S_{\text {raw }}$, for each case can be defined as 13:

$$
\begin{aligned}
& S_{\text {raw }}^{H e}=S_{\mathrm{R}}^{H e}+S_{\text {stray }}+S_{\text {dark }} \\
& S_{\text {raw }}^{O x}=S_{\mathrm{R}}^{O x}+S_{\text {stray }}+S_{\text {dark }}
\end{aligned}
$$

Here, $S_{\mathrm{R}}$ is the Rayleigh intensity, $S_{\text {stray }}$ is the stray light reflected from the surroundings and $S_{\text {dark }}$ is the dark noise of the camera. From these two equations, we see that the raw signals have contributions from Rayleigh scattering, stray light and 
dark current. Since Rayleigh signal is proportional to the scattering cross section $\sigma$, it follows

$$
S_{\mathrm{R}}^{H e}=\frac{\sigma^{H e}}{\sigma^{O x}} S_{\mathrm{R}}^{O x} .
$$

Substituting Eq. (3) into Eq. (1) and solving simultaneously with Eq. 22, we recover:

$$
S_{\text {stray }}=\left[\left(S_{\text {raw }}^{H e}-S_{\text {dark }}\right)-\frac{\sigma^{H e}}{\sigma^{O x}}\left(S_{\text {raw }}^{O x}-S_{\text {dark }}\right)\right]\left[1-\frac{\sigma^{H e}}{\sigma^{O x}}\right]^{-1}
$$

A simpler approach sometimes used is to assume that the images collected with helium flooding the imaging region contain negligible Rayleigh signal (i.e. $S_{\mathrm{R}}^{H e} \approx 0$ ). Although the scattering cross section of helium is approximately 100 times smaller compared to the oxidizer mixture, $\left(S_{\text {raw }}^{O x}-S_{\text {dark }}\right)$ was hundreds of counts in the reported system and so the helium signal was not negligible. The contribution of multiply-scattered light has been ignored, and secondary scatter of Rayleigh photons from surrounding surfaces was managed by placing a matte black wall around the burner at a distance of approximately $300 \mathrm{~mm}$. This wall serves to absorb many such photons, and scatter the remainder diffusely, strongly reducing the chance of their collection by the camera lens. Future work could employ structured illumination techniques to attempt to account for these sources of interference. Shot-to-shot laser energy fluctuation was assessed by calculating the average signal in a uniform region at the laser input edge of each frame in an oxidizer image set (30 columns wide, with constant temperature and composition). The standard deviation of these per-frame values was below $2 \%$. Energy correction was therefore not applied. The laser beam shape and geometrical/spectral/spatial collection efficiency factors were simultaneously dealt with by normalization: that is, taking the ratio between an averaged Rayleigh image of pure oxidizer and a Rayleigh image of the flame under test. The resulting Rayleigh ratio, $R_{R}$, is found from

$$
R_{R}=\frac{S_{\mathrm{R}}^{\text {Ox-avg }}}{S_{\mathrm{R}}^{\text {Flame }}}=\frac{\left(S_{\mathrm{raw}}-S_{\mathrm{stray}}-S_{\mathrm{dark}}\right)_{\mathrm{Ox}}}{\left(S_{\mathrm{raw}}-S_{\mathrm{stray}}-S_{\mathrm{dark}}\right)_{\mathrm{Flame}}} .
$$

Note that this Rayleigh ratio has the opposite behavior to Rayleigh signal; Rayleigh scattering is weak at high temperatures but the Rayleigh ratio has instead a large value. Image quality was quantified by determining the signal-to-noise ratio, SNR, of the cold and hot regions of the flame. For the cold region, this was determined by calculating the mean and standard deviation of pixel values in a uniform region of co-flowing gas (500x100 pixels at the edge of the image), and taking their ratio. For the hot region, pixel values were taken from column 244 in a laminar flame image, corresponding to a region which gives a nearly uniform and maximal Rayleigh ratio [37, and processed the same way. This calculation was repeated for all frames in a laminar flame data set, resulting in an average cold SNR of 36 and an average hot SNR of 13 . The stability of the laser sheet profile was assessed by examining a uniform region in an oxidizer image set. For each image, 30 columns were averaged together in order to reduce the relative strength of shot noise and characterize the laser intensity as a function of row number. From these data, the laser intensity at any given location was calculated to vary from image to image by less than $3 \%$. This analysis indicated that the laser beam profile was relatively stable and that average profile correction applied by normalization against the oxidizer image would suffice. The linearity of the camera response was verified following the procedure described in 20]. 
The statistics of the experimental data were obtained by taking the mean and standard deviation of the data in time providing an average and fluctuation value at each pixel location.

\section{Numerical Approach}

In the following, the simulation procedure of the laminar flame is briefly outlined in Sec. 3.1. with further details given in [17, 22, 33]. The modeling approach for the turbulent flame is explained in Sec. 3.2 Finally, the numerical approach for generating the Rayleigh signal is discussed in Sec. 3.3

All reactive simulations were performed applying a GRI-MECH 3.0 mechanism [50. This choice was made after a sensitivity study was performed comparing four different mechanisms and the results are presented in appendix C. It was shown that the influence of the kinetic mechanism is small in the considered flames and thus the widely-used GRI-MECH 3.0 mechanism was adopted.

\subsection{Laminar Flame}

The laminar simulation is performed as a detailed calculation evaluating the species source terms, diffusion and radiation terms directly. The governing transport equations for the density $\rho$, the velocity $\boldsymbol{u}$, the total enthalpy $h$ and the species mass fractions $Y_{i}$ are

$$
\begin{aligned}
\frac{\partial \rho}{\partial t}+\nabla \cdot(\rho \boldsymbol{u}) & =0 \\
\frac{\partial}{\partial t}(\rho \boldsymbol{u})+\nabla \cdot(\rho \boldsymbol{u u}) & =-\nabla p+\nabla \cdot \boldsymbol{\tau}+\rho \boldsymbol{g}, \\
\frac{\partial}{\partial t}(\rho h)+\nabla \cdot(\rho \boldsymbol{u} h) & =-\nabla \cdot \sum_{i=1}^{I} h_{i} \boldsymbol{j}_{i}+\nabla \cdot(\lambda \nabla T)-\nabla \cdot \boldsymbol{q}_{R}, \\
\frac{\partial}{\partial t}\left(\rho Y_{i}\right)+\nabla \cdot\left(\rho \boldsymbol{u} Y_{i}\right) & =-\nabla \cdot \boldsymbol{j}_{i}+R_{i},
\end{aligned}
$$

where $p$ is the pressure, $\boldsymbol{\tau}$ is the laminar momentum flux, $\boldsymbol{g}$ is gravity, $R_{i}$ is the chemical source term, $-\nabla \cdot \boldsymbol{q}_{R}$ is the radiative source term and $\boldsymbol{j}_{i}$ is the laminar diffusive mass flux, respectively. The diffusive flux is modeled using mixture-averaged diffusion coefficients [6] with a correction velocity to ensure mass conservation. Transport contributions due to the Soret effect are included using EGLib [14] and are discussed in the results section below. Different radiation modeling approaches are investigated. The most simple and widely employed optically thin model (OTM) with a gray absorption coefficient fit based on RADCAL simulations [19] is used here. This approach usually yields satisfactory results in standard air-combustion 3 and is thus also suggested by the TNF workshop [1. As a second model, the P1 approximation 34] combined with the weighted sum of gray gases (WSGG) 21] using the weights from 24] is used. The latter has already been successfully applied in oxy-fuel combustion [17.

Laminar simulations are performed with an in-house solver [17, 22, 33] based on OpenFOAM ${ }^{\circledR}$ 2.1. The inner jet is assumed to have a Hagen-Poiseuille velocity profile and the coflow velocity is set to a block profile. The computational domain includes 
$1 \mathrm{~mm}$ of the inlet tubes and extends to a height of $150 \mathrm{~mm}$ with a radius of $55 \mathrm{~mm}$. A 2D axisymmetric mesh with 50,000 hexahedral cells is used after grid-independence was ensured.

\subsection{Turbulent Flame}

The turbulent simulations use the flamelet/progress variable approach [40] within the Large Eddy Simulation framework. Transport of mass and momentum is performed by solving the Favre-filtered governing equations for the momentum $\bar{\rho} \widetilde{\boldsymbol{u}}$ and the density $\bar{\rho}$. Closure is achieved by means of an eddy viscosity hypothesis and the sub-grid turbulence, including the computation of the eddy viscosity $\mu_{\mathrm{t}}$, is evaluated with the sigma model [35], applying a fixed model constant, $C_{\sigma}=1.3$.

The FPV approach requires an additional transport equation to be solved for the conserved scalar mixture fraction $\widetilde{Z}$. Further, an equation is required for the nonnormalized progress variable $\widetilde{Y_{C}}$. Based on the assumption that the sub-grid scales are locally homogeneous and in equilibrium, an algebraic equation for $\overline{Z^{\prime \prime 2}}$ is solved [40]

$$
\begin{gathered}
\frac{\partial \bar{\rho} \widetilde{Z}}{\partial t}+\nabla \cdot(\bar{\rho} \widetilde{\mathbf{u}})=\nabla \cdot\left(\bar{\rho}\left(D+D_{t}\right) \nabla \widetilde{Z}\right) \\
\widetilde{Z^{\prime \prime 2}}=C_{\widetilde{Z^{\prime \prime 2}}} \Delta^{2}(\nabla \widetilde{Z})^{2} \\
\frac{\partial \bar{\rho} \widetilde{Y}_{C}}{\partial t}+\nabla \cdot\left(\bar{\rho} \widetilde{\mathbf{u}} \widetilde{Y}_{C}\right)=\nabla \cdot\left(\bar{\rho}\left(D+D_{t}\right) \nabla \widetilde{Y}_{C}\right)+\widetilde{\dot{\omega}}_{Y_{C}},
\end{gathered}
$$

where $D_{t}$ denotes the turbulent diffusivity calculated with a turbulent Schmidt-number $S c_{t}=\nu_{t} / D_{t}=0.4$ 26, 42, $D$ is the molecular diffusivity, $\Delta$ the filter width calculated from the cube root of the cell volume and the modeling coefficient is assumed to be $C_{\widetilde{Z^{\prime \prime 2}}}=0.13[15]$.

The thermo-chemical state is retrieved from flamelet look-up tables. For laminar nonpremixed flamelets the corresponding equations for temperature and species mass fractions are solved in the mixture fraction space [39]. When differential diffusion effects are considered, this leads to more complex flamelet equations [41. An in-house flamelet solver is used, which also performs the mapping from the scalar dissipation rate to the normalized progress variable $C=Y_{C} / Y_{C, e q}$. The filtered density function (FDF) -integration is performed by applying a presumed $\beta$-FDF for the mixture fraction and a $\delta$-FDF for the normalized progress variable, assuming statistical independence [40. For the FPV model, the choice of the progress variable is crucial [23, 36, 44. In this particular flame, the single species of $\mathrm{H}_{2} \mathrm{O}$ was found to obtain a good mapping of the scalar dissipation rate to the normalized progress variable and thus $\widetilde{Y}_{C}=\widetilde{Y}_{\mathrm{H}_{2} \mathrm{O}}$. The retrieval from the flamelet look-up table uses a multivariate interpolation. The interpolation weights are determined using a recursive $n$-dimensional multi-linear interpolation method optimized for memory placement and computational efficiency [57].

The simulations are performed applying a unity Lewis-number approach. In the turbulent flame with $\mathrm{Re}=18000$ the near field is expected to be influenced by differential diffusion mechanisms. However, the simulations applying a unity Lewis-number in comparison with constant, but non-unity Lewis-numbers showed a good agreement with the experimental data in the considered regions. Thus the regions recorded in the experiment and compared to the simulation results appear less influenced by differential diffusion effects. A corresponding analysis is presented in appendix E 
The turbulent flame is simulated with an in-house solver based on the OpenFOAM ${ }^{\circledR} 2.1$ framework. The jet velocity inflow boundary is based on a simulation of the pipe flow in order to generate turbulent time-dependent inflow profiles. These profiles are transported into the computational domain of the actual jet from the jet boundary. The coflow velocity is set to a block profile. In order to validate this procedure, a cold flow of an air-air mixture was investigated experimentally and numerically. The experiments measured the instantaneous axial flow velocity using hot-wire anemometry, which was processed to obtain the mean velocity $\langle\tilde{u}\rangle$ and its variance $\left\langle u^{\prime 2}\right\rangle$ as described in Appendix D.1 The comparison of the numerical and the experimental results is shown in Appendix D.2. Good agreement was obtained justifying the use of the same procedure for the reactive flow simulation. The computational domain is a rectilinear grid of the size $30 \times 30 \times 150 \mathrm{~mm}$ at the jet inlet and enlarges to $60 \times 60 \times 150 \mathrm{~mm}$ at the outlet with a grid resolution of $64 \times 64 \times 300$ cells, globally refined towards the nozzle. The reported statistics are collected after running the simulation for 3 flow through times (FTT) and for a period of 12 FTT by time-integration. Azimuthal averaging was performed in addition. The overall numerical setup for reactive LES was successfully used for a partially premixed DME flame [43] without extinction and reignition events and is thus also applicable for the flame investigated here.

\subsection{Numerical Evaluation of the Rayleigh Signal}

As described above, the predicted Rayleigh ratio is directly compared to the experimental Rayleigh ratio. In the following, the procedure of generating this signal in the numerical simulation is described. The Rayleigh intensity can be calculated with

$$
S_{R}=I_{\text {Laser }} C_{\exp } N \sum_{i} X_{i} \sigma_{R i}
$$

where $I_{\text {Laser }}$ is the laser intensity, $C_{\exp }$ the experimental factor, which includes the calibration corresponding to the experimental setup, $N$ the total number density, $X_{i}$ the mole fraction and $\sigma_{R i}$ the Rayleigh cross section of the considered species $i$. In order to compare the signal to the experimental data, the same ratio as described by Eq. (5) is calculated here. However, in the numerical simulation the reflected stray light $S_{\text {stray }}$ as well as the dark noise of the camera $S_{\text {dark }}$ are zero. The numerically calculated ratio can thus be simplified to

$$
R_{R}=\frac{S_{\mathrm{R}}^{O x}}{S_{\mathrm{R}}^{\text {Flame }}} .
$$

Introducing Eq. (13) leads to

$$
R_{R}=\frac{T}{T_{\text {Ox }}} \frac{\sum_{i=1}^{n_{\text {Ox }}} X_{i} \sigma_{R i}}{\sum_{j=1}^{n_{\text {Flame }}} X_{j} \sigma_{R j}} .
$$

The numerical Rayleigh ratio is thus only a function of the local species mole fractions and the local temperature as well as the oxidizer species mole fractions and temperature and can be evaluated based on the local and instantaneous numerical results.

The constant species-specific cross sections for the main species $\mathrm{CO}_{2}, \mathrm{CO}, \mathrm{O}_{2}, \mathrm{CH}_{4}$, $\mathrm{H}_{2} \mathrm{O}, \mathrm{H}_{2}$ and some significant intermediates $\mathrm{OH}, \mathrm{O}, \mathrm{H}, \mathrm{C}_{2} \mathrm{H}_{4}, \mathrm{C}_{2} \mathrm{H}_{6}, \mathrm{C}_{3} \mathrm{H}_{8}, \mathrm{C}_{2} \mathrm{H}_{2}$ and $\mathrm{CH}_{3}$ are evaluated following [16]. The remaining species mass fraction is below $0.5 \%$ 
and its contribution to the Rayleigh cross section is likely to be small. Further, Fuest et al. [16] discussed the temperature dependence of the species cross section and found them to vary by less than $1 \%$ in his DME flame, whereas a variation of up to $2 \%$ towards low temperatures was found in [54]. By employing the relationships suggested in 54 we estimate that the cross section inaccuracy with increasing temperature is not more than $2 \%$.

In the laminar case, the species and temperature are fully available and the evaluation of the Rayleigh ratio can thus be performed based on this detailed simulation without any further assumption at each location in the flow field.

In the turbulent case, the species and temperature data are known from the laminar flamelet results. Using the same procedure as for the other scalars, the Rayleigh ratio is calculated based on the FDF-integrated instantaneous laminar flamelet data. In order to ensure a consistent comparison with the experimental Rayleigh ratio, we use a nondensity weighted FDF, which can be obtained from the Favre-filtered FDF as described in [40].

\section{Results and Discussion}

In the following, the comparison between the experimentally recorded Rayleigh ratio and the numerically calculated Rayleigh ratio is shown for the laminar flame in Sec. 4.1 and for the turbulent flame in Sec. 4.2 For the laminar flame, a detailed study was performed. Due to the availability of the stationary and fully resolved species and temperature profiles, the influence of different modeling approaches and assumptions for diffusion and radiation on the Rayleigh ratio can be studied and analyzed in detail. In contrast, in the turbulent flame the statistics of the LES results are compared to the experimental data. The direct comparison of the experimentally recorded and numerically predicted Rayleigh ratio enables us to investigate and post-process the instantaneous data in the entire recorded 2D domain as shown for the time-averages and the distribution function below.

\subsection{Laminar Flame}

First, the flame structure is analyzed based on the experimental and the numerical Rayleigh ratio. Figure 4 shows the overall temperature field and the Rayleigh ratio on the left side and a comparison between the numerical data of temperature and Rayleigh ratio in the two measurement locations on the right side. The flame structure shown was simulated with the numerical approach found to give the best agreement, namely applying the GRI-MECH 3.0 mechanism, including thermal radiation via the P1 approximation with WSGG and also considering the Soret effect. Further details are given below where the influence of different modeling approaches is shown in more detail. Differences between the shapes of the contours of temperature and Rayleigh ratio can be seen, indicating that the transformation from Rayleigh signal to temperature is not trivial for the general case of non-constant scattering cross sections of the mixture. The traditional approach of assuming a constant effective Rayleigh cross section is insufficient here and assumptions of the species mass fractions would be required. Consequently, the present direct comparison of Rayleigh ratio is highly advantageous, 

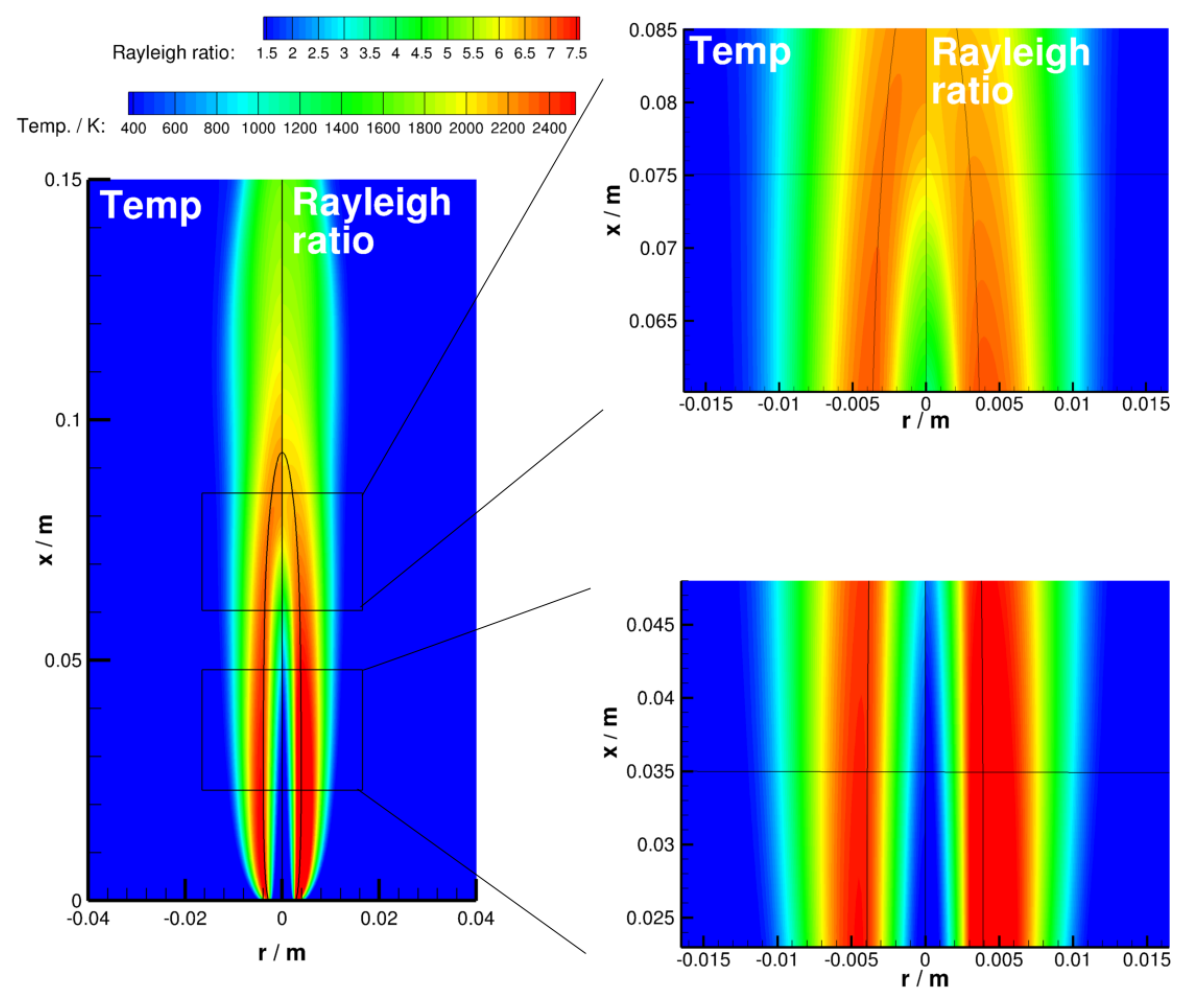

Fig. 4: Contour plot of the numerical approach with the Soret effect and the P1 radiation model using the GRI-MECH 3.0 mechanism. In each plot, numerical data of temperature is always on the left and of Rayleigh ratio always on the right. The entire flame is shown in the left column and the two locations where measurements were performed are marked. The corresponding numerical data are shown on the right. The slices extracted for detailed comparison are marked as horizontal lines. The stoichiometric mixture fraction $Z_{\text {st }}=0.418$ is also highlighted.

omitting such assumptions and simplifications but still allowing the experimental and numerical results to be compared.

Further, Figs. 5 and 6 show a comparison of the experimental Rayleigh ratio and the numerical simulation with different radiation modeling approaches as described in Sec 3.1. also including an adiabatic simulation. From left to right, Figure 5 illustrates the experimental data, the simulation with the P1 approach, the simulation with the OTM approach and an adiabatic simulation, respectively. Large differences between the different radiation modeling approaches can be found, which are most evident in the higher measurement location, when the temperature decreases. The $\mathrm{P} 1$ radiation model yields a good agreement with the experimental data both in structure and absolute values. On the other hand, the OTM approach significantly underestimates the Rayleigh ratio. Finally, the adiabatic simulation overestimates the Rayleigh ratio tremendously, as expected. In order to quantify this comparison, a single line in each measurement window is extracted and compared in Fig. 6, namely at heights of $35 \mathrm{~mm}$ 

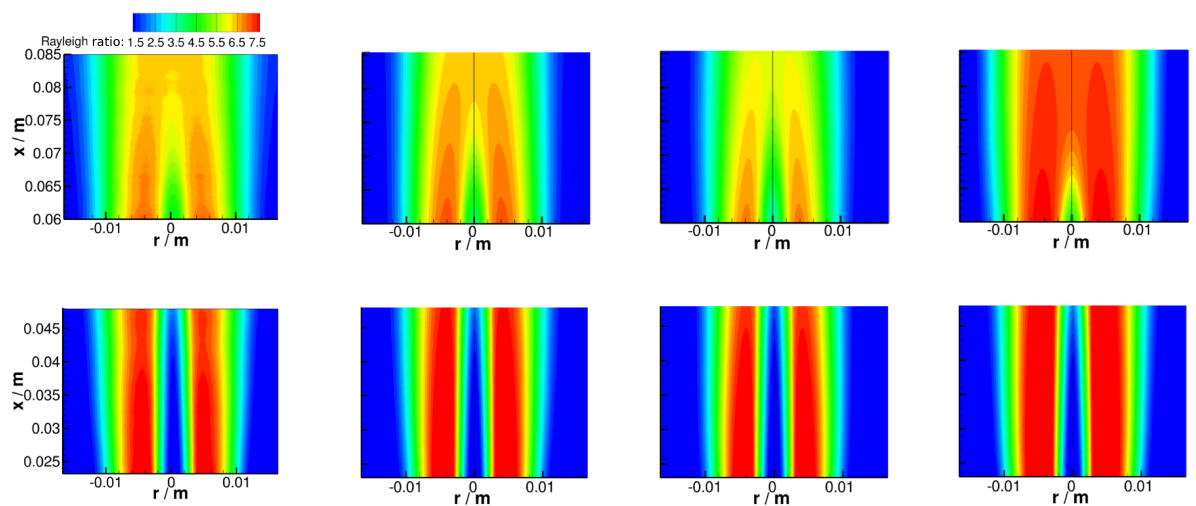

(a) Exp.

(b) Sim. P1

(c) Sim. OTM

(d) Sim. ad

Fig. 5: Side-by-side comparison of the experimental and the numerical Rayleigh ratio with different radiation models. Top: $60-85 \mathrm{~mm}$ height and bottom: $23-48 \mathrm{~mm}$ height.

and $75 \mathrm{~mm}$. The Rayleigh ratio, the temperature and the species mass fractions of $\mathrm{CO}_{2}$, $\mathrm{H}_{2}$ and $\mathrm{H}_{2} \mathrm{O}$ are shown along these two slices. As already indicated, large differences are evident for the different radiation modeling approaches. The adiabatic simulation overestimates the Rayleigh ratio significantly in both measurement locations, whereas the OTM approach underestimates the signal. According to Eq. 15, this is expected as the temperature is underestimated with the OTM approach [51]. However, at a height of $35 \mathrm{~mm}$ the OTM approach is still within the experimental uncertainty, calculated from the standard deviation of 1000 single shots for each pixel, which is no longer true at a height of $75 \mathrm{~mm}$. In general, the $\mathrm{P} 1$ model agrees well with the experimental data. As discussed in [17 this is due to the oxy-fuel conditions and therewith the occurence of optically denser regions. The temperature profiles exhibit similar trends as the Rayleigh ratio. Differences between the different models are distinct. Looking at the main species profiles shows that the different radiation models have only a minor influence. Differences can be depicted, which are more pronounced further downstream, especially for $\mathrm{CO}_{2}$. The observed differences in the Rayleigh ratio as a result of different radiation models are thus dominated by temperature change rather than species profile changes.

Finally, Figs. 7 and 8 show the comparison of the experimental Rayleigh ratio and the numerical simulation investigating the influence of the Soret effect. From left to right, Fig. 7 shows the experimental data and the simulations with and without the Soret effect, respectively. The influence of the Soret effect on the Rayleigh ratio is much smaller compared to the choice of the radiation model but still notable. This is reasonable because the Soret effect mostly redistributes the species and temperature profiles with little change of the absolute temperature level. As discussed during the radiation model comparison, the latter has a much higher influence on the Rayleigh ratio. However, differences can be seen, which are most evident along the burner central axis. Here, neglecting the Soret effect would lead to a signal maximum further upstream. This phenomenon is quantified again along two lines in Fig. 8 for the Rayleigh ratio, the temperature and the species $\mathrm{CO}_{2}, \mathrm{H}_{2} \mathrm{O}$ and $\mathrm{H}_{2}$. As mentioned above and looking at the Rayleigh ratio, the largest differences are found near the burner central axis 

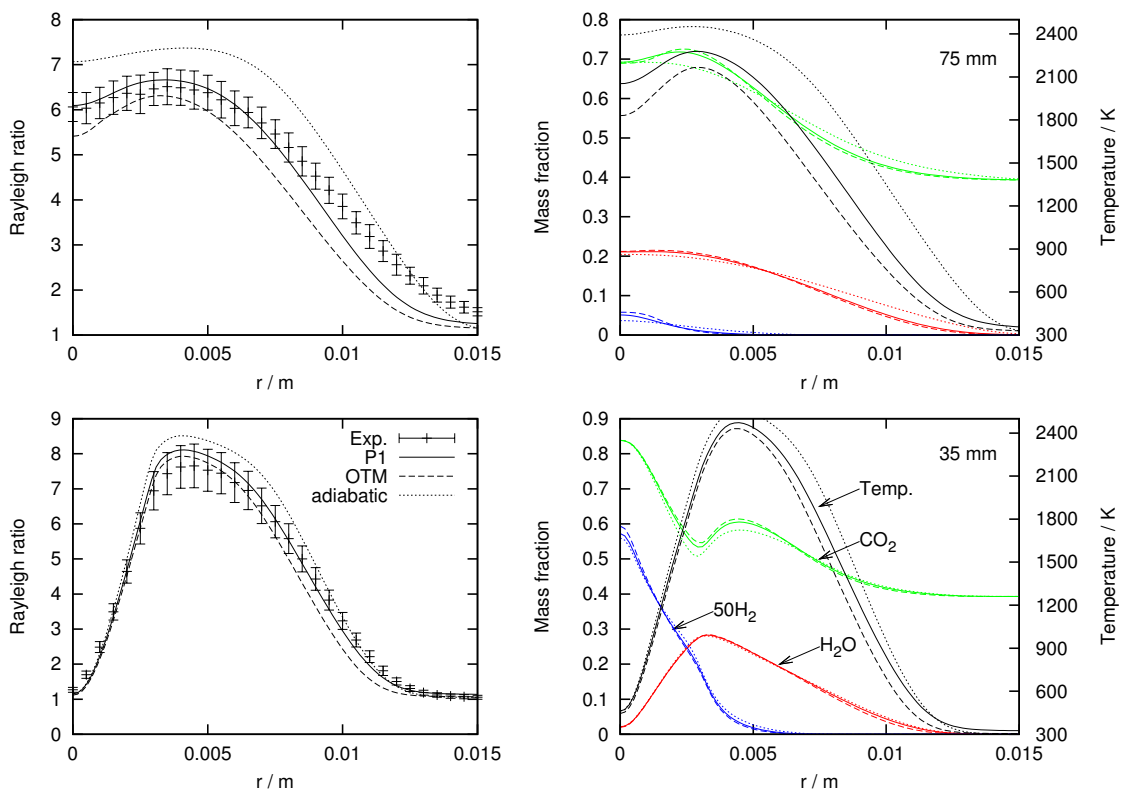

Fig. 6: Comparison of the experimental Rayleigh ratio and the numerical simulation (left) and the temperature and species profiles of $\mathrm{CO}_{2}, \mathrm{H}_{2}$ and $\mathrm{H}_{2} \mathrm{O}$ of the simulation (right) both with different radiation models along a radial line; top: $75 \mathrm{~mm}$ height and bottom: $35 \mathrm{~mm}$ height.
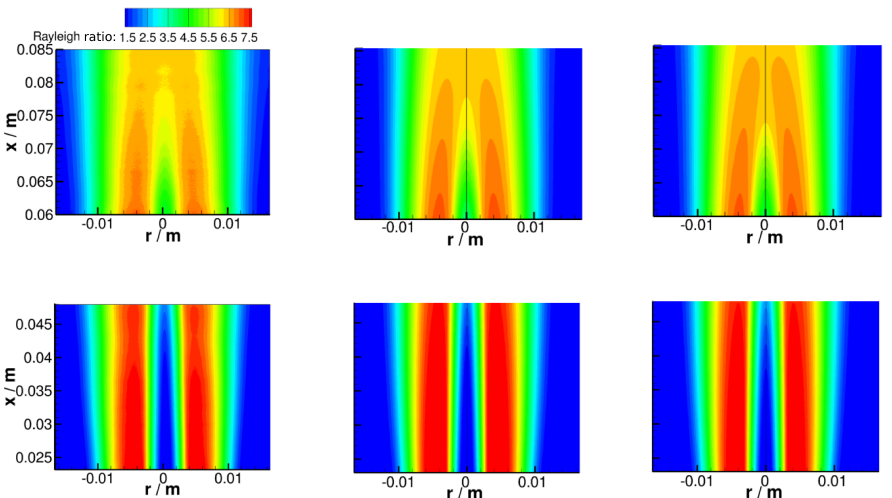

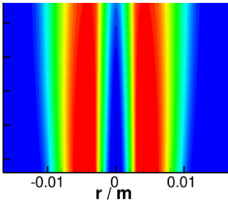

(b) Sim. w Soret

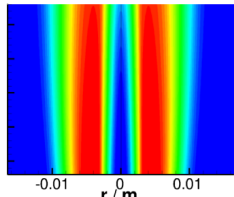

(c) Sim. w/o Soret

(a) Exp.

Fig. 7: Side-by-side comparison of the experimental and the numerical Rayleigh ratio with and without the Soret effect. Top: 60-85 mm height and bottom: $23-48 \mathrm{~mm}$ height. 

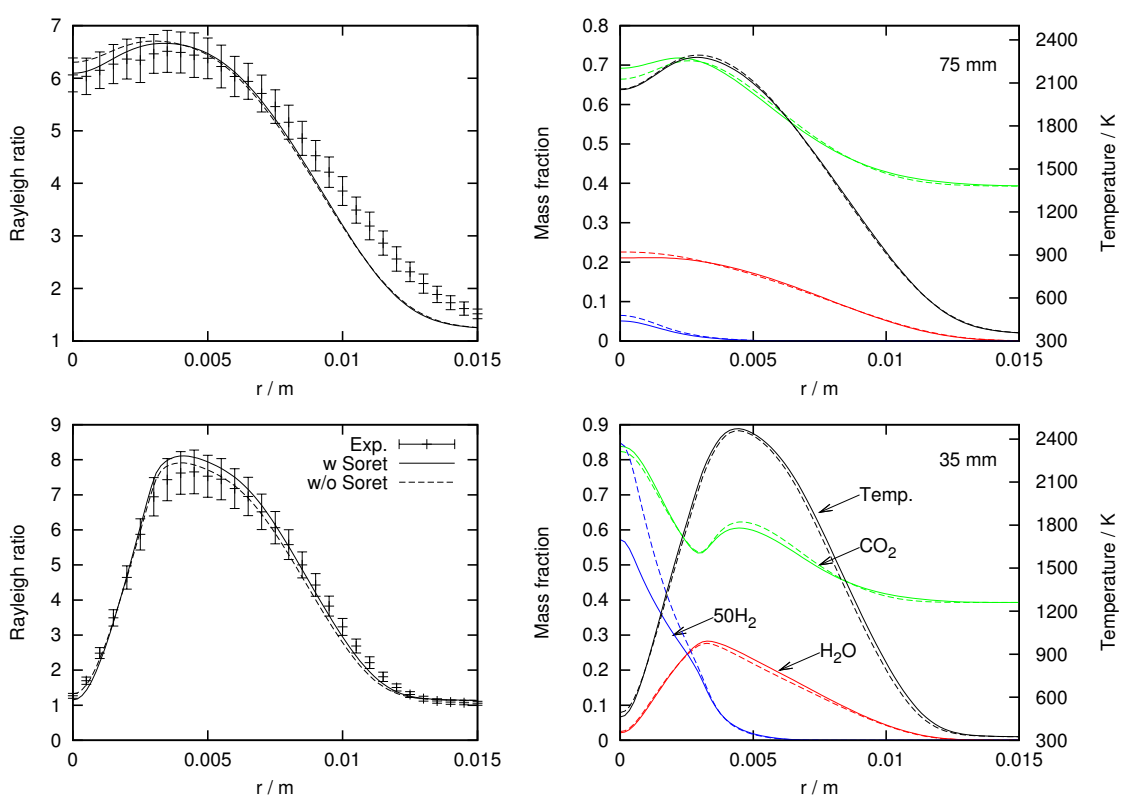

Fig. 8: Comparison of the experimental Rayleigh ratio and the numerical simulation (left) and the temperature and species profiles of $\mathrm{CO}_{2}, \mathrm{H}_{2}$ and $\mathrm{H}_{2} \mathrm{O}$ of the simulation(right) both with and without the Soret effect along a radial line; top: $75 \mathrm{~mm}$ height and bottom: $35 \mathrm{~mm}$ height.

further downstream in the flame. However, differences in the overall profile can also be seen at a height of $35 \mathrm{~mm}$. On the other hand, the species exhibit larger differences in their profiles compared to the different radiation models. Differences can be found near the burner central axis, but also along the complete radius, which is especially true for $\mathrm{CO}_{2}$ and $\mathrm{H}_{2}$. The influence of these differences on the Rayleigh ratio is moderate. However, a slightly better agreement is obtained with the Soret effect. In contrast, the temperature profiles are almost congruent for the different diffusion models (small differences are found in the height of $35 \mathrm{~mm}$ ). Thus, small (species) changes are more visible in Rayleigh ratio data rather than in the temperature data. The Rayleigh ratio is also a suitable metric for diffusion model validation.

The investigation of the laminar flame showed that comparison of the experimental and numerical Rayleigh ratio is feasible. Both structure and absolute values show good agreement. Furthermore, the direct comparison enables the evaluation of modeling assumptions. The choice of radiation model influences the Rayleigh ratio mostly due to the change in the temperature level. Changes in the species profiles and thus also of the effective Rayleigh cross section lead only to a local and modest change of the Rayleigh ratio and are less pronounced. The influence of the Soret effect is less dominant but still observed in the Rayleigh ratio. Consequently, direct comparison of Rayleigh ratio has been shown to be sensitive to small changes which might otherwise be masked when using the traditional method of comparing derived temperature. 


\subsection{Turbulent Flame}

The turbulent flame is also analyzed by direct comparison of the Rayleigh ratio. Here, we use the time-averaged filtered Rayleigh ratio $\left\langle\bar{R}_{R}\right\rangle$ and the time-averaged resolved variance $\left\langle\bar{R}_{R}^{\prime 2}\right\rangle$ or its RMS value $\sqrt{\left\langle\bar{R}_{R}^{\prime 2}\right\rangle}$, respectively. Further, the probability density function (PDF) of the Rayleigh ratio is evaluated. When investigating the instantaneous data as performed when computing the PDFs, the entire domain that was experimentally recorded can be used and appropriate and interesting locations can be identified as shown later. By contrast, a purely experimental approach to recovering temperature would be restricted to the point or line where simultaneous Raman/Rayleigh measurements had been recorded.

Figure 9 shows a part of the computational domain, including the time-averaged temperature and the time-averaged Rayleigh ratio. In addition, instantaneous snapshots of the flame in the three different measurement locations are illustrated in Fig. 9, comparing the experiments and the numerical simulation for qualitative illustration. The differences between the time-averaged temperature and the time-averaged Rayleigh ratio are smaller compared to the laminar flame. In general, the flame structure is captured well since the broadening of the high temperature zone agrees well. In the first region, a thin layer region with a high Rayleigh ratio can be found. Further downstream, the flame shape (as indicated by Rayleigh ratio values larger than 1.0) becomes broader and wider ranges of a high Rayleigh ratio occur. In the highest measurement window close to the flame tip, the flame zone broadens even further.

The Rayleigh ratio mean and variance are compared for each location in Fig. 10 showing the experiments on the left and the simulation results on the right side. The overall structure of the Rayleigh ratio agrees well in all three measurement locations. The broadening of the mean Rayleigh ratio further downstream is captured well as is the bimodal shape of the signal variance. In addition, the general thickness of the signal with values larger than 1.0 is captured accurately. However, it is evident that the peak values of the mean Rayleigh ratio are underestimated by at most $7 \%$ in the simulation, whereas the signal variance is overestimated by at most $5 \%$.

In order to further quantify these results, the Rayleigh ratio is extracted at a representative height in each of the measurement windows and plotted as a function of radius for the heights of 35, 55 and $110 \mathrm{~mm}$ in Fig. 11 on the left. As noted above, a slight underestimation of the mean Rayleigh ratio can be seen in the height of $35 \mathrm{~mm}$. Here, the numerical signal also yields a narrower shape compared to the experimental profile. However, further downstream, the numerical signal broadens and both the profile and the absolute values agree well with the experimental results. Similarly, the RMS data has its peak slightly closer to the burner central axis in the simulation compared to the experiments. Further downstream, the profiles agree very well. In general, a good agreement between the statistical values is obtained for the Rayleigh ratio. Processing the Rayleigh ratio in turbulent simulations within the flamelet framework thus yields good agreement between experiments and simulations. In this approach, the Rayleigh ratio is treated as a solution of the laminar flamelet and is fully integrated in the LES-FPV approach. An FDF integration accounts for subgrid contributions. Thus, the non-linear behavior of the Rayleigh ratio, combining the influence of the temperature and the species mole fractions, is captured well within the LES-FPV model.

In order to further investigate the statistics of the Rayleigh ratio recorded in the experiments and calculated in the FPV approach, Fig. 11 on the right shows the PDF of 


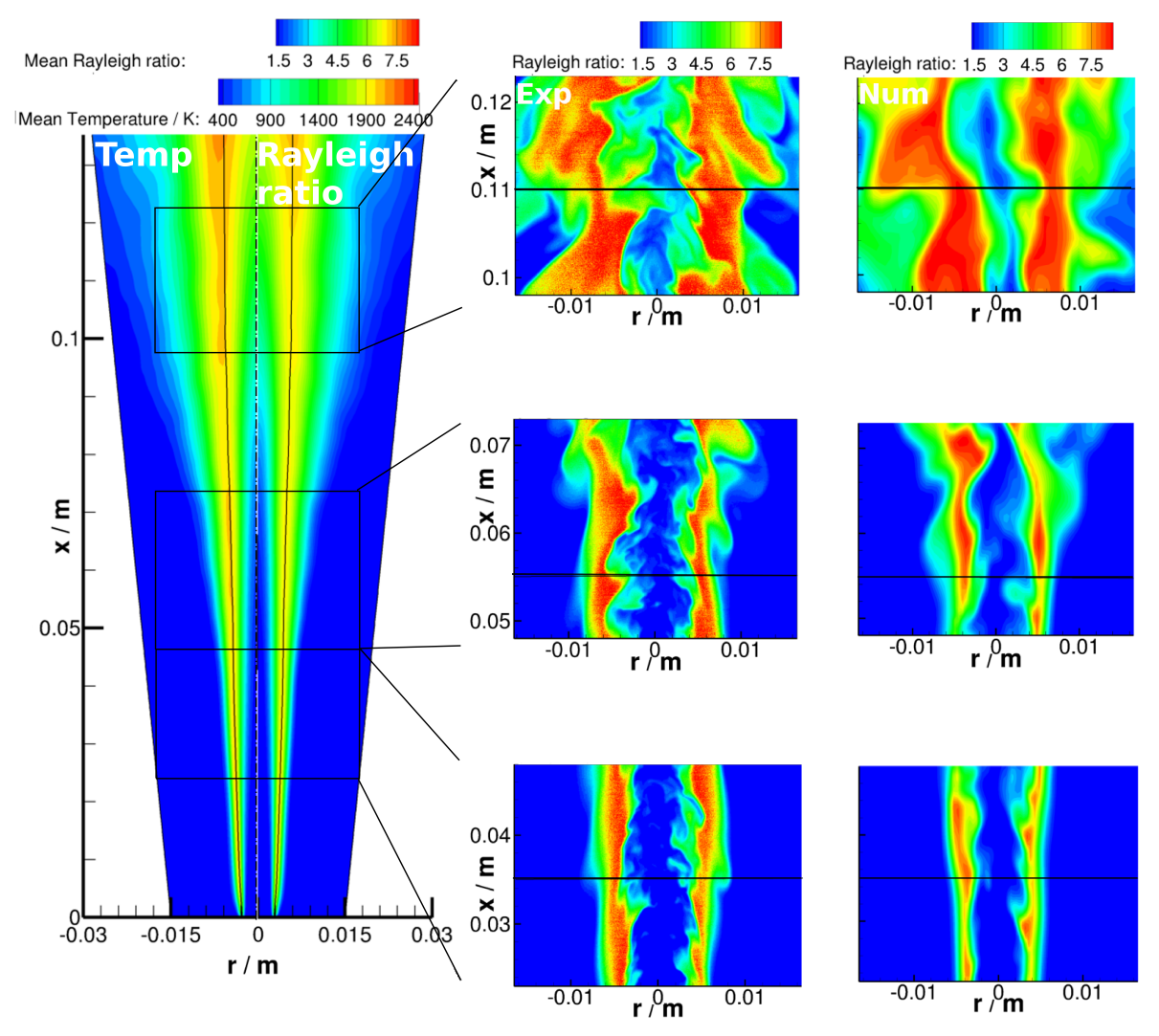

Fig. 9: Contour plot of the time-averaged temperature and the time-averaged Rayleigh ratio of the flame and side-by-side comparison of the instantaneous experimental (left) and the numerical Rayleigh ratio (right) in top: $98-123 \mathrm{~mm}$ height, middle: $48-73 \mathrm{~mm}$ height and bottom: 23-48 mm height. The slices extracted for detailed comparison are marked as horizontal lines in each measurement window. The stoichiometric mixture fraction $Z_{\text {st }}=0.418$ is marked.

the Rayleigh ratio along the same slices. This representation was previously used for Rayleigh signal analysis by McManus et al. 32. The distribution of the Rayleigh ratio is shown at each point along the radius by using color to represent the PDF values. It is worth noting that we are free in the choice of the location where the PDFs should be investigated since the Rayleigh measurements are recorded in $2 \mathrm{D}$ and the predicted Rayleigh ratio is numerically available in the entire calculation domain. The PDF of the numerical simulation is calculated based on 15,000 snapshots, whereas the experimental PDF uses 1000 snapshots. Overall, good agreement is observed. In general, the range of fluctuations increases further downstream and thus the maximum values of the PDF decrease. Further, at the heights of 35 and $55 \mathrm{~mm}$ the peak values of the PDF are locally very narrow, close to the peak of $\left\langle\bar{R}_{R}\right\rangle$. In contrast, at a height of $110 \mathrm{~mm}$ a much broader range of maximum values of the PDF is found. The maximum PDF 

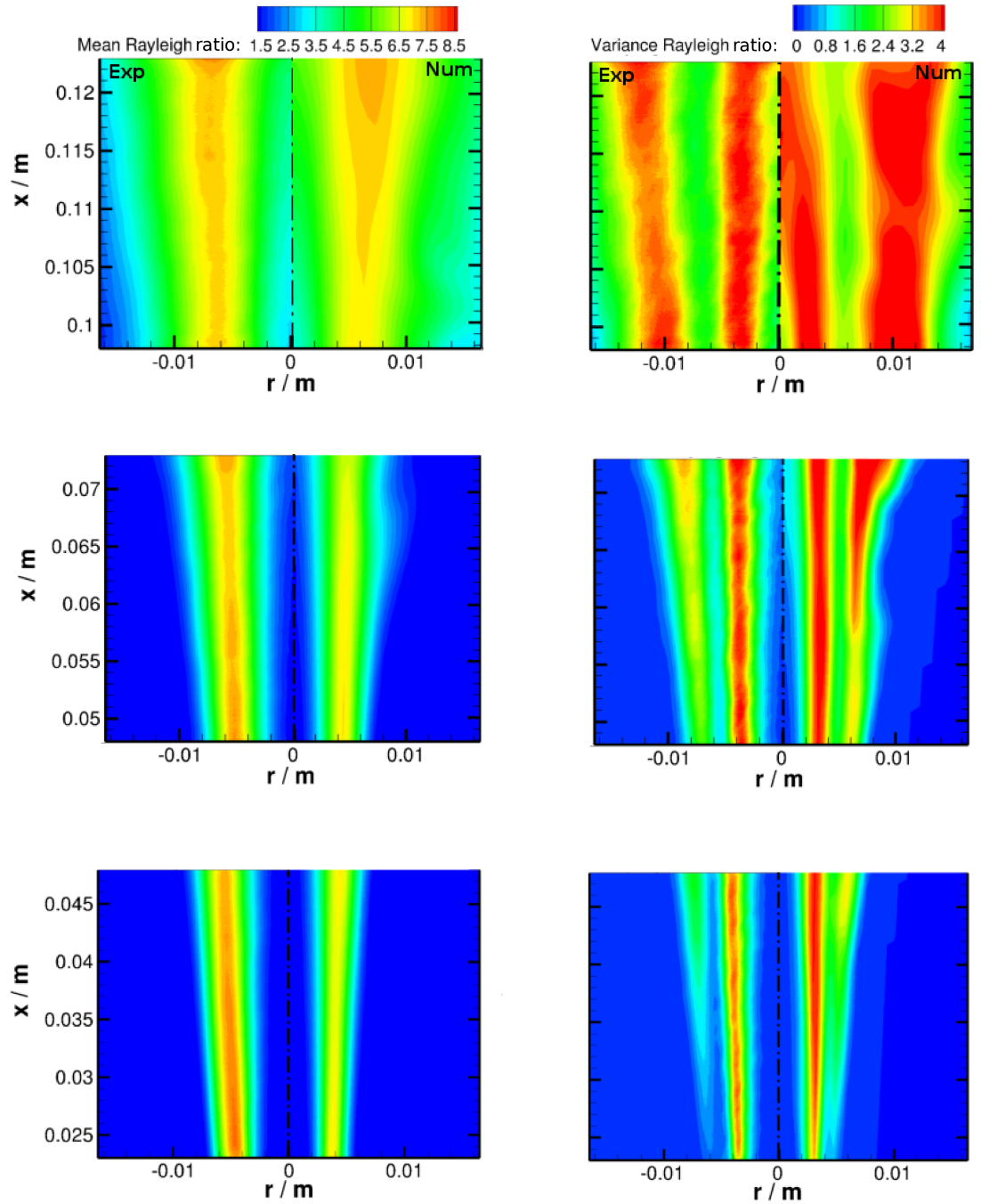

Fig. 10: Side-by-side comparison of the time-averaged experimental and numerical Rayleigh ratio (left) and the experimental and numerical Rayleigh ratio variance (right) in top: $98-123 \mathrm{~mm}$ height, middle: $48-73 \mathrm{~mm}$ height and bottom: $23-48 \mathrm{~mm}$ height.

values are found varying from 7 to 8.5 , and also over a broader range of radii, from $r=5 \mathrm{~mm}$ to $r=10 \mathrm{~mm}$.

To aid quantitative interpretation of Fig. 11 individual PDFs are shown for six different flame locations in Fig. 12 Here, we extract the distribution for a given height at the location of the maximum mean signal, $\left\langle\bar{R}_{R}\right\rangle$, illustrated in the left column and at the point of the highest signal fluctuation, $\sqrt{\left\langle\bar{R}_{R}^{\prime 2}\right\rangle}$, illustrated in the right column. When looking in the left column of Fig. 12, the maximum PDF value decreases further downstream and the PDF shape becomes broader, with a tail towards lower Rayleigh 

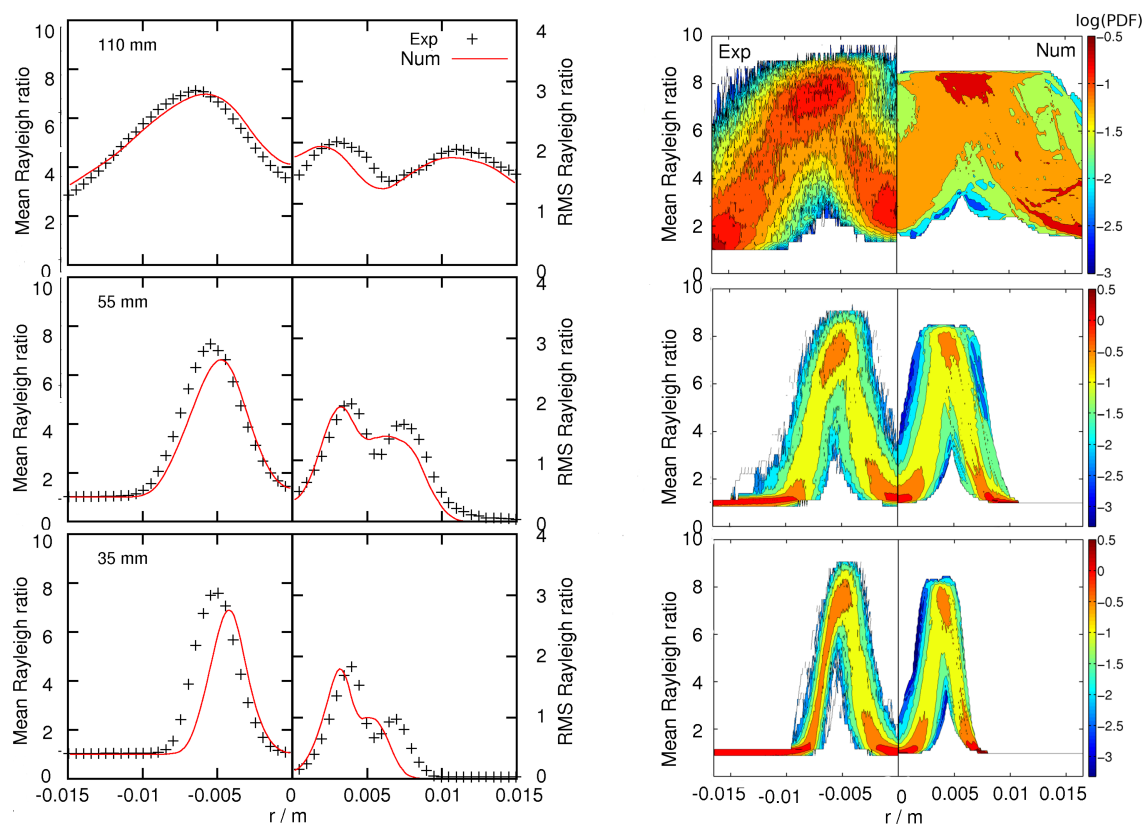

Fig. 11: Side-by-side comparison of the experimental data and the numerical simulation. Left: mean and RMS Rayleigh ratio, right: the PDF of the Rayleigh ratio in top: 110 $\mathrm{mm}$ height, middle: $55 \mathrm{~mm}$ height and bottom: $35 \mathrm{~mm}$ height.

ratio values. On the other hand, the PDFs from regions of highest fluctuations (right column) are very similar at all three heights. The PDFs show almost constant values over a broad range of signal values, indicating that the maximum variance does not change significantly at the different heights. Comparing the experimental bars to the red numerical line in the left column shows a distribution slightly shifted towards smaller Rayleigh ratio values at a height of $35 \mathrm{~mm}$, but very good agreement is found further downstream, consistent with the $\left\langle\bar{R}_{R}\right\rangle$ profile along the radius at the same height shown in Fig. 11. The right-hand column of fluctuation PDFs shows that the simulation captures this aspect well.

In summary, we found the statistics of the predicted Rayleigh ratio to agree well with the experimental Rayleigh ratio. A comparison of the 2D recorded experimental data along a line in each of the three measurement locations yielded a good qualitative agreement. The quantitative comparison of the time-averaged Rayleigh ratio and its RMS revealed a slight shift near the jet nozzle but agreed well further downstream. This was also confirmed by the investigation of the PDFs where the instantaneous data of experiment and numerics were used for the comparison. We thus found the direct comparison of the Rayleigh ratio to be both feasible and a suitable metric for improved model validation especially in turbulent flames. Note that in general, instantaneous Rayleigh-inferred temperature data must be supported by composition information which would normally be derived from Raman scattering. This requirement restricts such measurements to 1D. Employing our method, model validation is enhanced because an accurate and meaningful comparison may be drawn over the whole 2D domain. 

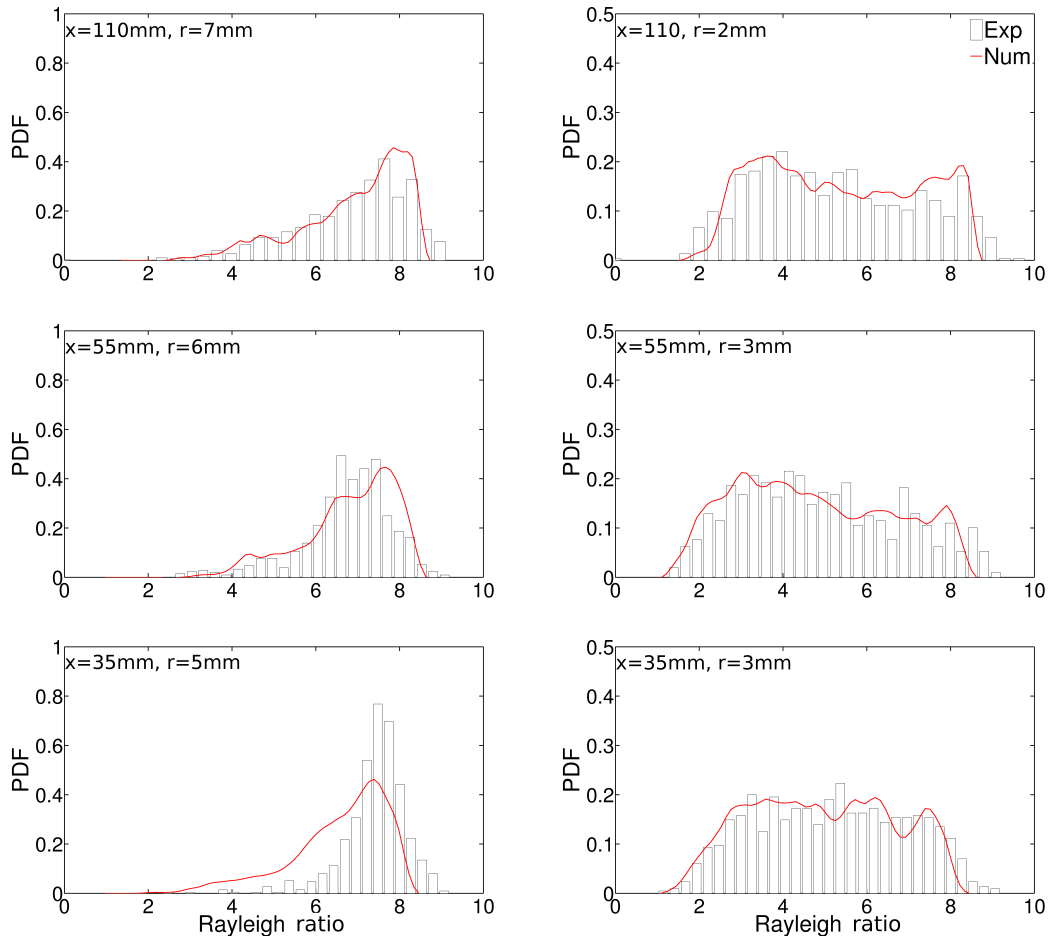

Fig. 12: PDF of the Rayleigh ratio extracted at 6 different flame locations. Left: showing data from location of peak mean Rayleigh ratio and right: data from location of strongest fluctuations; top: $110 \mathrm{~mm}$ height, middle: $55 \mathrm{~mm}$ height and bottom: $35 \mathrm{~mm}$ height.

\section{Conclusions}

In the present study a laminar and a turbulent oxy-fuel jet flame were investigated experimentally and numerically with special emphasis on the comparison of the Rayleigh ratio. The Rayleigh ratio was measured in both flame setups, carefully correcting for stray light and dark noise by capturing and processing images of pure oxidizer and a helium flow. Equivalent Rayleigh ratios were derived from the numerical species data and temperature.

The comparison between the two different Rayleigh ratios in the laminar flame showed generally very good agreement both in position and absolute value. This success verified the capability of the concept, removing inaccuracies and assumptions traditionally present in experimental measurements of temperature using Rayleigh scattering. Calculating the Rayleigh ratio in laminar flames is thus feasible. Further, the influence of different modeling approaches for radiation and diffusion showed the sensitivity of the Rayleigh ratio to these models. It was found that the radiation model choice does influence the absolute signal values significantly mostly due to changes in the temperature rather than change in composition. P1 radiation modeling with WSGG was shown to yield the best results for this laminar oxy-fuel flame. The Soret effect was also investi- 
gated, which mainly influences the species distribution. Differences were strongest for $\mathrm{H}_{2}$, but also evident for $\mathrm{CO}_{2}$. However, the effect on the Rayleigh ratio was moderate since the absolute temperature level was not changed. Overall, the importance of the Soret effect to Rayleigh ratio was demonstrated in this configuration. Thus, model validation can be performed based on a Rayleigh signal alone, yielding improved sensitivity and accuracy compared to traditional Rayleigh-inferred temperature.

For the LES of the turbulent flame, the Rayleigh ratio prediction was integrated into the flamelet/progress variable approach. Here, the Rayleigh ratio was calculated based on the laminar flamelet results and FDF-integrated in order to obtain the Reynolds-filtered values required for the fully-coupled LES. Good agreement between the experimental and numerical 2D field data was obtained. The flame structure based on the Rayleigh ratio agreed well. Some differences were found in mean and RMS values in the most upstream region. In order to investigate the statistics of the Rayleigh ratio recorded in the experiments in comparison with the statistics of the predicted Rayleigh ratio calculated in the FPV approach, the PDF of the Rayleigh ratio was investigated. The PDF of the Rayleigh ratio clearly showed the appearance of the flame. Very narrow and steep PDFs were found closer to the jet inlet, whereas further downstream a much broader PDF was present. We thus showed that a comparison of the PDFs generated in an arbitrary location within the $2 \mathrm{D}$ measurement domain can be a successful approach for turbulent flame validation.

To summarize, we have demonstrated that experimental Rayleigh signals can be directly compared with numerically predicted values for model validation of laminar and turbulent oxy-fuel combustion. This approach was able to discern relatively subtle differences between different models which might be masked by traditional Rayleighderived temperature measurements. In such traditional diagnostics, the composition of the gas must either be measured (leading to 1D Raman/Rayleigh experiments) or known a priori (leading to synthetic fuel/oxidizer specifications). Our approach enables accurate model validation to be performed across an entire $2 \mathrm{D}$ field of view whilst using a realistic fuel-oxidizer combination and reducing experimental complexity.

Acknowledgements The authors would like to gratefully acknowledge the financial support by the Federal Ministry of Education and Research of Germany in framework of Virtuhcon (project number 03Z2FN11).

\section{References}

1. International workshop on measurement and computation of turbulent flames. http://www.sandia.gov/TNF/abstract.html (2012)

2. Barlow, R.S.: Laser diagnostics and their interplay with computations to understand turbulent combustion. Proceedings of the Combustion Institute 31(1), 49 75 (2007)

3. Barlow, R.S., Karpetis, A.N., Frank, J.H., Chen, J.Y.: Scalar profiles and NO formation in laminar opposed-flow partially premixed methane/air flames. Combustion and Flame 127, 2102-2118 (2001)

4. Baukal, C.E., Gebhart, B.: Oxygen-enhanced/natural gas flame radiation. Int. J. Heat Mass Transfer 40(11), 2539 - 2547 (1997) 
5. Bergmann, V., Meier, W., Wolff, D., Stricker, W.: Application of spontaneous raman and rayleigh scattering and $2 \mathrm{D}$ LIF for the characterization of a turbulent CH4/H2/N2 jet diffusion flame. Applied Physics B 66(4), 489-502 (1998)

6. Bird, R.B., Stewart, W.E., Lightfoot, E.N.: Transport Phenomena. 2nd edn. Wiley (2012)

7. Böhm, B., Brübach, J., Ertem, C., Dreizler, A.: Experiments for combustion-LES validation. Flow, Turbulence and Combustion 80(4), 507-529 (2008)

8. Connelly, B., Bennett, B., Smooke, M., Long, M.: A paradigm shift in the interaction of experiments and computations in combustion research. Proceedings of the Combustion Institute 32(1), 879 - 886 (2009)

9. Coriton, B., Zendehdel, M., Ukai, S., Kronenburg, A., Stein, O.T., Im, S.K., Gamba, M., Frank, J.H.: Imaging measurements and LES-CMC modeling of a partially-premixed turbulent dimethyl ether/air jet flame. Proceedings of the Combustion Institute (2014)

10. Dibble, R.W., Hollenbach, R.E.: Laser rayleigh thermometry in turbulent flames. Proceedings of the Combustion Institute 18(1), 1489-1499 (1981)

11. Ditaranto, M., Hals, J.: Combustion instabilities in sudden expansion oxy-fuel flames. Combustion and flame 146(3), 493-512 (2006)

12. Du, D.X., Axelbaum, R.L., Law, C.K.: The influence of carbon dioxide and oxygen as additives on soot formation in diffusion flames. Proceedings of the Combustion Institute 23, 1501-1507 (1991)

13. Dunn, M.J.: Finite-rate chemistry effects in turbulent premixed combustion. Ph.D. thesis, University of Sydney (2008)

14. Ern, A., Giovangigli, V.: Fast and accurate multicomponent transport property evaluation. Journal of Computational Physics (120), 105-116 (1995)

15. Forkel, H., Janicka, J.: Large-eddy simulation of a turbulent hydrogen diffusion flame. Flow, Turbulence and Combustion 65(2), 163-175 (2000)

16. Fuest, F., Barlow, R.S., Chen, J.Y., Dreizler, A.: Raman/Rayleigh scattering and CO-LIF measurements in laminar and turbulent jet flames of dimethyl ether. Combustion and Flame 159(8), 2533-2562 (2012)

17. Garten, B., Hunger, F., Messig, D., Stelzner, B., Trimis, D., Hasse, C.: Detailed radiation modeling of a partial-oxidation flame. International Journal of Thermal Sciences 87, 68-84 (2015)

18. Glarborg, P., Bentzen, L.L.B.: Chemical effects of a high CO2 concentration in oxy-fuel combustion of methane. Energy \& Fuels 22(1), 291-296 (2007)

19. Grosshandler, W.L.: RADCAL: A Narrow-Band Model for Radiation Calculations in a Combustion Environment. Tech. rep., NIST technical note 1402 (1993)

20. Hain, R., Köhler, C., Tropea, C.: Comparison of CCD, CMOS and intensified cameras. Experiments in Fluids 42(3), 403-411 (2007)

21. Hottel, H.C., Sarofim, A.F.: Radiative Transfer. McGraw-Hill, New York (1967)

22. Hunger, F., Stelzner, B., Trimis, D., Hasse, C.: Flamelet-modeling of inverse rich diffusion flames. Flow, Turbulence and Combustion 90, 833-857 (2013)

23. Ihme, M., Shunn, L., Zhang, J.: Regularization of reaction progress variable for application to flamelet-based combustion models. Journal of Computational Physics 231(23), 7715 - 7721 (2012)

24. Kangwanpongpan, T., França, F.H.R., da Silva, R.C., Schneider, P.S., Krautz, H.J.: New correlations for the weighted-sum-of-gray-gases model in oxy-fuel conditions based on HITEMP 2010 database. International Journal of Heat and Mass Transfer 55, 7419-7433 (2012) 
25. Kee, R.J., Rupley, F.M., Miller, J.A., Coltrin, M.E., Grcar, J.F., Meeks, E., Moffat, H.K., Lutz, A.E., Dixon-Lewis, G., Smooke, M.D.: Chemkin-pro release 15082 (2008)

26. Kempf, A., Flemming, F., Janicka, J.: Investigation of lengthscales, scalar dissipation, and flame orientation in a piloted diffusion flame by LES. Proceedings of the Combustion Institute 30(1), 557 - 565 (2005)

27. Kim, H.K., Kim, Y., Lee, S.M., Ahn, K.Y.: Studies on combustion characteristics and flame length of turbulent oxy-fuel flames. Energy Fuels 21(3), 1459-1467 (2007)

28. Kohse-Höinghaus, K.: Laser techniques for the quantitative detection of reactive intermediates in combustion systems. Prog. Energy Combust. Sci. 20(3), 203-279 (1994)

29. Krishnan, S.S., Saini, M.K., Zheng, Y., Gore, J.P.: Radiation properties of oxygenenhanced normal and inverse diffusion flames. J. Heat Transfer 134(2), 022701 (2012)

30. Linow, S., Dreizler, A., Janicka, J., Hassel, E.P.: Measurement of temperature and concentration in oxy-fuel flames by raman/rayleigh spectroscopy. Measurement Science and Technology 13(12), 1952 (2002)

31. Mazas, A.N., Lacoste, D.A., Schuller, T.: Experimental and numerical investigation on the laminar flame speed of $\mathrm{CH} 4 / \mathrm{O} 2$ mixtures diluted with $\mathrm{CO} 2$ and $\mathrm{H} 2 \mathrm{O}$. ASME Conference Proceedings 2010(43970), 411-421 (2010)

32. McManus, T.A., Papageorge, M.J., Fuest, F., Sutton, J.A.: Spatio-temporal characteristics of temperature fluctuations in turbulent non-premixed jet flames. Proceedings of the Combustion Institute 35 (2014)

33. Messig, D., Hunger, F., Keller, J., Hasse, C.: Evaluation of radiation modeling approaches for non-premixed flamelets considering a laminar methane air flame. Combustion and Flame 160, 251-264 (2013)

34. Modest, M.: Radiative Heat Transfer. 3rd edn. Academic Press (2013)

35. Nicoud, F., Toda, H.B., Cabrit, O., Bose, S., Lee, J.: Using singular values to build a subgrid-scale model for large eddy simulations. Physics of Fluids 23(8), 085106 (2011)

36. Niu, Y.S., Vervisch, L., Tao, P.D.: An optimization-based approach to detailed chemistry tabulation: Automated progress variable definition. Combustion and Flame 160(4), 776 - 785 (2013)

37. Papageorge, M., McManus, T., Fuest, F., Sutton, J.: Recent advances in high-speed planar rayleigh scattering in turbulent jets and flames: increased record lengths, acquisition rates, and image quality. Applied Physics B 115(2), 197-213 (2014)

38. Park, J., Park, J.S., Kim, H.P., Kim, J.S., Kim, S.C., Choi, J.G., Cho, H.C., Cho, K.W., Park, H.S.: No emission behavior in oxy-fuel combustion recirculated with carbon dioxide. Energy Fuels 21(1), 121-129 (2007)

39. Peters, N.: Laminar Flamelet Concepts in Turbulent Combustion. Proceedings of the Combustion Institute 21, 1231-1250 (1986)

40. Pierce, C., Moin, P.: Progress-variable approach for large-eddy simulation of nonpremixed turbulent combustion. Journal of Fluid Mechanics 504, 73-97 (2004)

41. Pitsch, H., Peters, N.: A consistent flamelet formulation for non-premixed combustion considering differential diffusion effects. Combustion and Flame 114(1-2), 26 - 40 (1998)

42. Pitsch, H., Steiner, H.: Large-eddy simulation of a turbulent piloted methane/air diffusion flame (Sandia flame D). Physics of Fluids (1994-present) 12(10), 2541- 
$2554(2000)$

43. Popp, S., Hunger, F., Hartl, S., Messig, D., Coriton, B., Frank, J.H., Fuest, F., Hasse, C.: LES flamelet-progress variable modelling and measurements of a turbulent partially-premixed dimethyl ether jet flame. Combustion and Flame 162(8), 3016-3029 (2015)

44. Prüfert, U., Hartl, S., Hunger, F., Messig, D., Eiermann, M., Hasse, C.: A constrained control approach for the automated choice of an optimal progress variable for chemistry tabulation. Flow Turbulence and Comustion 94(3), 593-617 (2015)

45. Rasmussen, C.L., Rasmussen, A.E., Glarborg, P.: Sensitizing effects of nox on ch4 oxidation at high pressure. Combustion and Flame 154(3), 529 - 545 (2008)

46. Samaniego, J.M., Mantel, T.: Fundamental mechanisms in premixed turbulent flame propagation via flame-vortex interactions: Part I: experiment. Combustion and flame 118(4), 537-556 (1999)

47. Scheffknecht, G., Al-Makhadmeh, L., Schnell, U., Maier, J.: Oxy-fuel coal combustion - a review of the current state-of-the-art. Int. J. Greenhouse Gas Control 5, Supplement 1(0), S16 - S35 (2011)

48. Schießl, R., Kaiser, S., Long, M., Maas, U.: Application of reduced state spaces to laser-based measurements in combustion. Proceedings of the Combustion Institute 32(1), $887-894$ (2009)

49. Sevault, A., Dunn, M., Barlow, R.S., Ditaranto, M.: On the structure of the near field of oxy-fuel jet flames using Raman/Rayleigh laser diagnostics. Combustion and Flame pp. $3342-3352$ (2012)

50. Smith, G.P., Golden, D.M., Frenklach, M., Moriarty, N.W., Eiteneer, B., Goldenberg, M., Bowman, C.T., Hanson, R.K., Song, S., William C. Gardiner, J., Lissianski, V.V., Qin, Z.: Gas research institute. http://www.me.berkeley.edu/gri_ mech/version30 (2012)

51. Stelzner, B., Hunger, F., Voss, S., Keller, J., Hasse, C., Trimis, D.: Experimental and numerical study of rich inverse diffusion flame structure. Proc. Combust. Inst. 34, 1045-1055 (2013)

52. Stelzner, B., Hunger, F., Laugwitz, A., Gräbner, M., Voss, S., Uebel, K., Schurz, M., Schimpke, R., Weise, S., Krzack, S., Trimis, D., Hasse, C., Meyer, B.: Development of an inverse diffusion partial oxidation flame and model burner contributing to the development of 3rd generation coal gasifiers. Fuel Processing Technology 110, 33-45 (2013)

53. Stepowski, D., Cabot, G.: Single-shot temperature and mixture fraction profiles by rayleigh scattering in the development zone of a turbulent diffusion flame. Combustion and Flame 88(3-4), 296 - 308 (1992)

54. Sutton, G., Levick, A., Edwards, G., Greenhalgh, D.: A combustion temperature and species standard for the calibration of laser diagnostic techniques. Combustion and Flame 147(1-2), $39-48$ (2006)

55. Wang, H., You, X., Joshi, A.V., Davis, S.G., Laskin, A., Egolfopoulos, F., Law, C.K.: High-temperature combustion reaction model of $\mathrm{H} 2 / \mathrm{CO} / \mathrm{C} 1-\mathrm{C} 4$ compounds. http://ignis.usc.edu/USC_Mech_II.htm (2007)

56. Wang, L., Endrud, N.E., Turns, S.R., D'Agostini, M.D., Slavejkov, A.G.: A study of the influence of oxygen index on soot, radiation, and emission characteristics of turbulent jet flames. Combustion science and technology 174(8), 45-72 (2002)

57. Weise, S., Hasse, C.: Reducing the memory footprint in large eddy simulations of reactive flows. Parallel Computing 49, 50-65 (2015) 
58. Zeißler, R.: Modellierung der Gasphasenreaktion bei der autothermen katalytischen Erdgasspaltung unter hohen Drücken. Dissertation, TU Bergakademie Freiberg (2005) 


\section{A OH-LIF Comparison in the Laminar Flame}

The comparison of the numerical OH-LIF signal (excitation with the $Q_{1}(8)$ line, spectrally integrated detection of fluorescence in the (0-0) and (1-1) band of the electronic $A^{2} \Sigma-X^{2} \Pi$ transition) is shown in the following. Note that the OH-LIF profiles are qualitative and are thus normalized. For detailed explanation refer to 43 .

Figure 13 shows the contour plots of normalized OH-LIF using different radiation models in the same measurement windows as the Rayleigh signal, c.f. Fig. 4 The P1 radiation model is taken to be the reference in this case, since it showed best agreement for the Rayleigh comparison. Figure 13 shows that the OTM radiation model leads to a generally narrower profile with lower maximum values while the adiabatic simulation broadens the distribution of OH-LIF and increases the peak signal level in the displayed regions.
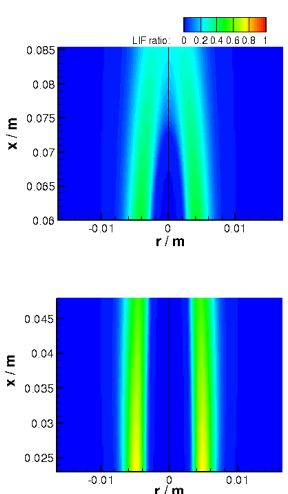

(a) Sim. P1
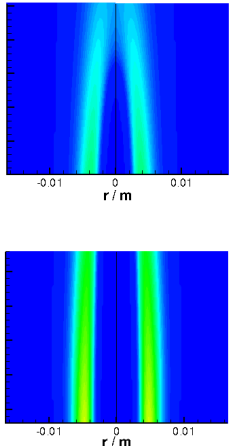

(b) Sim. OTM
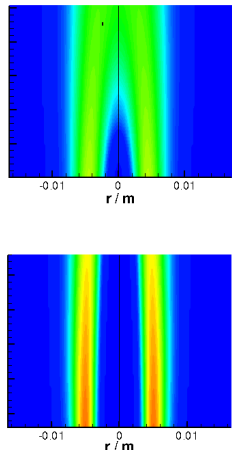

(c) Sim. ad.

Fig. 13: Side-by-side comparison of the numerical LIF ratio for two different radiation models and an adiabatic simulation. Top: 60-85 $\mathrm{mm}$ height and bottom: 23-48 $\mathrm{mm}$ height.

Quantitative evaluation of OH-LIF signal is not straight-forward and thus sometimes qualitative comparisons are performed that normalize the OH-LIF signal 7] 28, which must be mirrored in the simulation, e.g. 9, 22. Thus, Fig. 14 shows the comparison of the OH-LIF signal normalized to 1.0 at each height and for the three radiation models. The OH mass fraction is shown in addition for quantification. Similar trends as shown in the contour plots can be found. In the adiabatic simulation the OH-LIF profile is widened relative to the reference simulation and the corresponding $\mathrm{OH}$ mass fraction increases. The OTM radiation model obtains a narrower $\mathrm{OH}-\mathrm{LIF}$ profile and the $\mathrm{OH}$ mass fraction decreases. However, when looking at the normalized OH-LIF signal, these differences are less pronounced.

In summary, the OH-LIF measurement technique can also be used for radiation model analysis if the radiative heat loss is large enough. However, model validation is less effective since differences are smaller. Here, Rayleigh scattering is the better choice. 

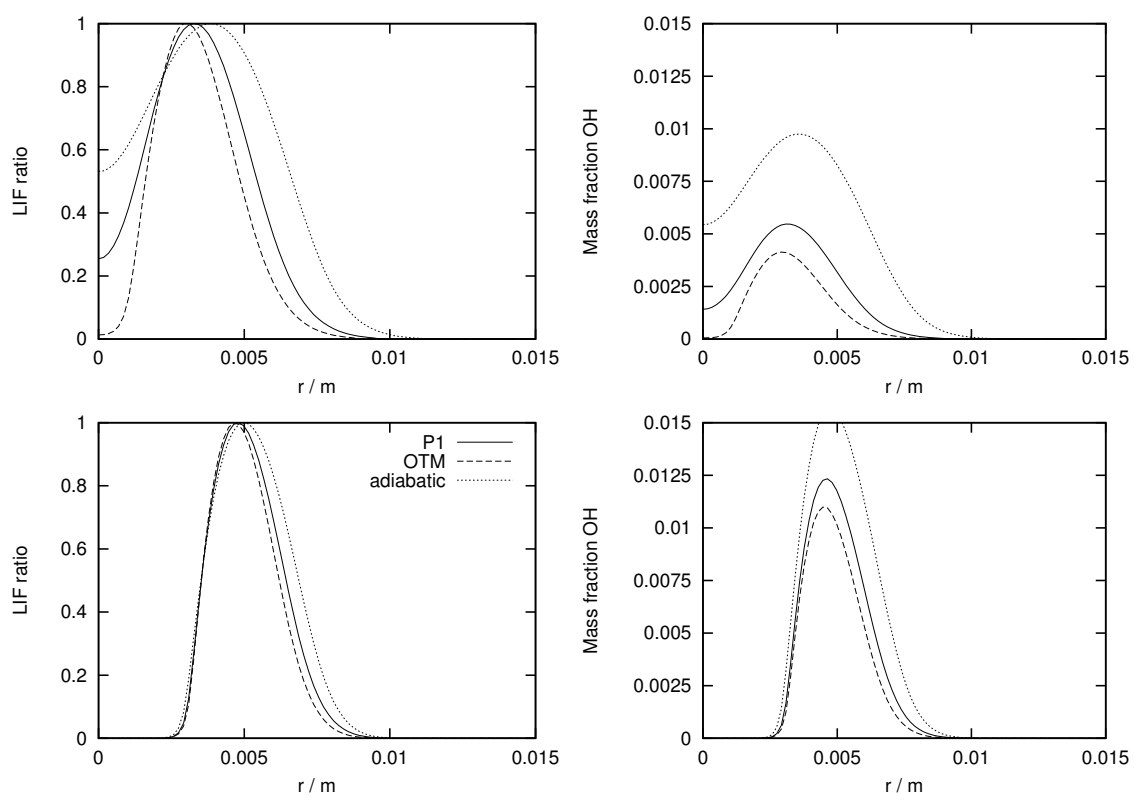

Fig. 14: Comparison of the numerical OH-LIF ratio (left) and the $\mathrm{OH}$ mass fraction (right) using 2 different radiation models and an adiabatic simulation along a radial slice in top: $75 \mathrm{~mm}$ height and bottom: $35 \mathrm{~mm}$ height.

\section{B Appearance of the Flame}

Figure 15 shows the appearance of the flame as well as the jet pipe and the coflow inlet. It further indicates the measurement windows and illustrates single snapshots of the recorded Rayleigh ratio. 


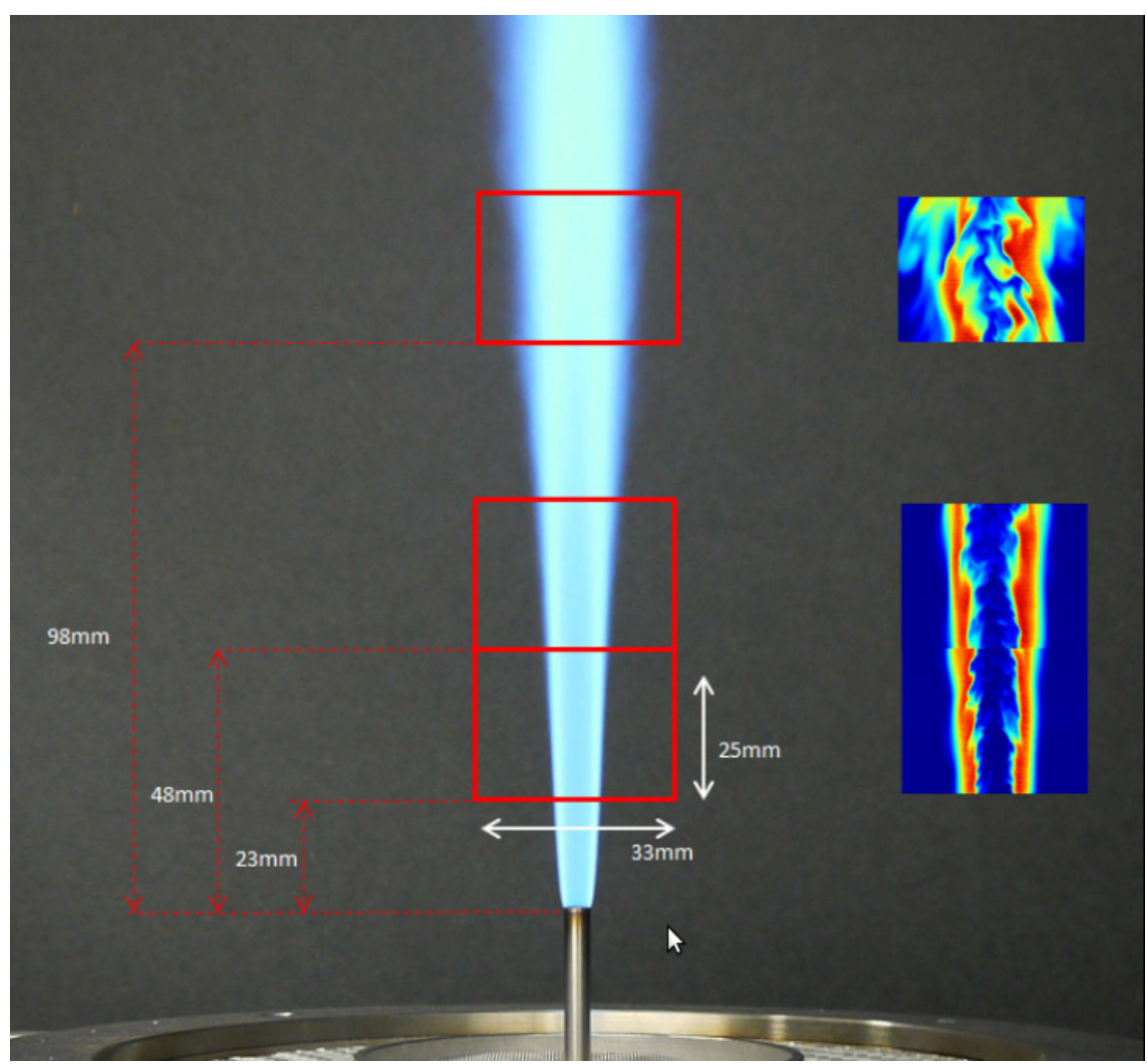

Fig. 15: Appearance of the flame in the experimental setup as well as the measurement windows and illustration of the Rayleigh ratio contour.

\section{Analysis of Different Reaction Mechanisms}

The influence of different kinetic mechanisms on the Rayleigh ratio is studied in the following. Here, several mechanisms, namely GRI-MECH 3.0 [50, a reduced GRI-MECH 3.0 [58, the mechanisms by Rasmussen et al. [5] and Wang et al. 55] are investigated and shown in Fig. 16 from left to right. Note that the mechanisms vary by their number of species and number of reactions. However, the mechanisms all contain chemical paths up to $\mathrm{C}_{2}$.

Differences between the different mechanisms are negligible when looking at the Rayleigh contour plots. More detailed knowledge can be obtained when quantifying the Rayleigh ratio as well as the main species along a single line as evaluated in Fig. 17 Some minor differences in the Rayleigh ratio as well as in the main species and temperature distribution can be seen. These differences are very small and are thus not considered any further here. GRI-MECH 3.0 is the standard chemical mechanism and this analysis shows no reason to choose anything different for this work. 

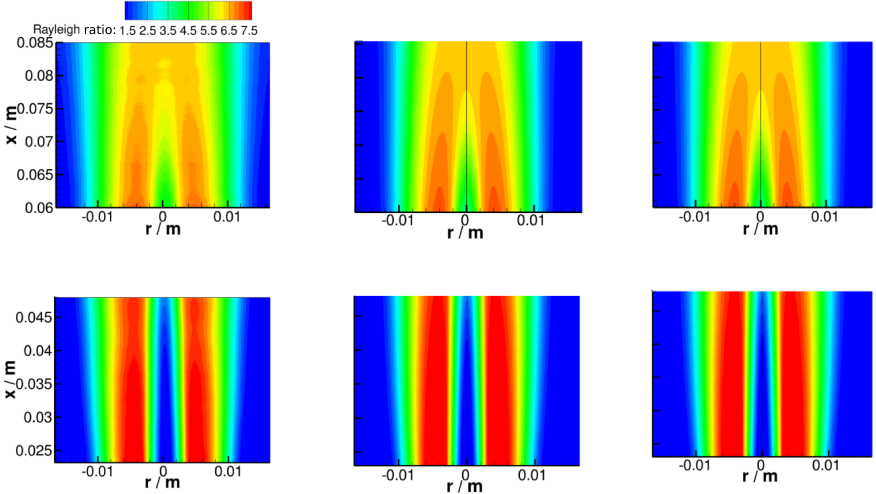

(a) Exp.

(b) Sim. GRI 3.0

(c) Sim. ATR
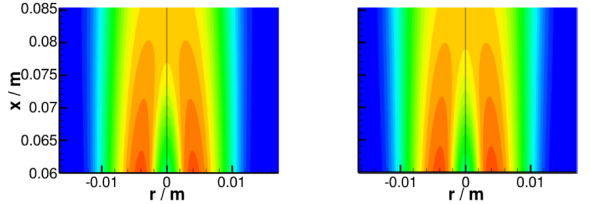

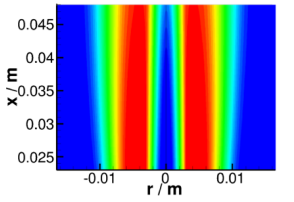

(d) Sim. Wang

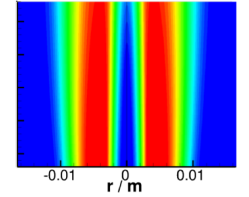

(e) Sim. Glaborg

Fig. 16: Side-by-side comparison of the experimental and the numerical Rayleigh ratio with 4 different reaction mechanisms. Top: 60-85 mm height and bottom: $23-48 \mathrm{~mm}$ height.

\section{Turbulent Cold Jet}

Since turbulent boundary conditions are very important for the simulation of turbulent flows and especially the jet break-up, the axial velocity profiles of a cold jet with the same conditions as in the flame were investigated experimentally and numerically.

\section{D.1 Hot-wire anemometry}

Velocity measurements were conducted with a hot-wire anemometer (HWA) probe using a DANTEC StreamLine 90N10 frame in conjunction with a 90C10 constant temperature anemometer (CTA) module. A single $1.25 \mathrm{~mm}$ long wire of $5 \mu \mathrm{m}$ diameter platinum-plated tungsten was first calibrated using a DANTEC calibrator unit. The unit is designed to calibrate a number of velocity ranges and in light of the intended bulk flow velocities, calibration for the range $0.5 \mathrm{~m} / \mathrm{s}-80 \mathrm{~m} / \mathrm{s}$ was chosen. A twenty point velocity calibration was carried out to establish a relation between CTA output and flow velocity, approximated through a 4th order polynomial curve fit. Temperature compensation was taken into account in both calibration and velocity profile measurements. 

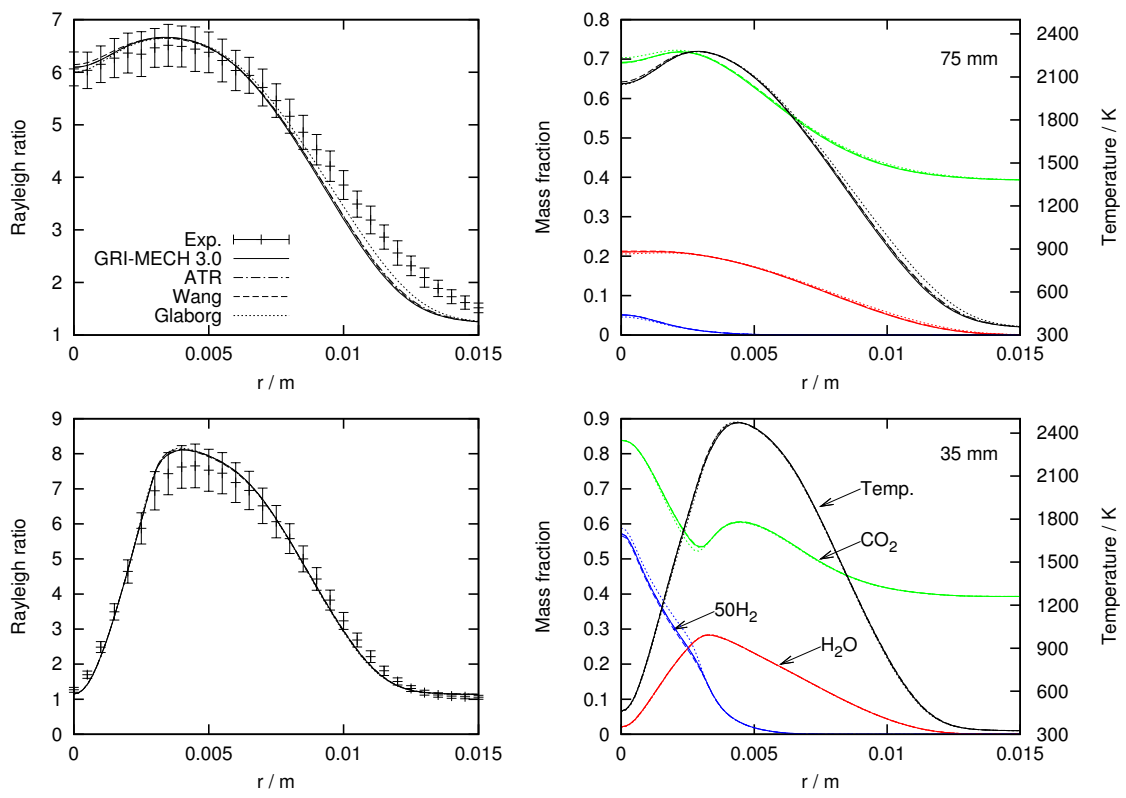

Fig. 17: Comparison of the experimental and the numerical Rayleigh ratio (left) and the temperature and species profiles of $\mathrm{CO}_{2}, \mathrm{H}_{2}$ and $\mathrm{H}_{2} \mathrm{O}$ of the simulation (right) using 4 different kinetic mechanisms along a radial slice in top: $75 \mathrm{~mm}$ height and bottom: $35 \mathrm{~mm}$ height.

Table 2: Flow conditions and measured bulk velocity

\begin{tabular}{|c|c|}
\hline Re & Mass Flow Rate Jet \\
\hline 15,000 & $8.5537 \cdot 10^{-4} \mathrm{~kg} / \mathrm{s}$ \\
\hline 18,000 & $1.0264 \cdot 10^{-3} \mathrm{~kg} / \mathrm{s}$ \\
\hline
\end{tabular}

Measurements in isothermal jets of air were obtained under the conditions presented in Tab. 2 and velocities were measured $1,3,10$ and $20 \mathrm{~mm}$ above the jet exit, along the radial distance of $\pm 15 \mathrm{~mm}$ from the nozzle center. The calculated bulk velocity (for the measurement at $1 \mathrm{~mm}$ above the nozzle) stems from the mass flow controllers while the measured bulk velocity is the integration of the HWA data, and demonstrates excellent agreement. The probe was traversed in steps of $0.5 \mathrm{~mm}$ near the center $( \pm 10 \mathrm{~mm})$ and the remaining steps were done with $1 \mathrm{~mm}$ separation.

\section{D.2 Numerical Simulation}

According to the measurements described above, a cold flow simulation was performed with the same procedure described in Sec. 3.2 in order to compare the turbulent flow field. The results of the 4 different measurement heights are shown in Fig. 18

The comparison shows good agreement of the mean velocity field in all measurement heights indicating that the procedure of prescribing the velocity field with an a priori LES pipe flow simulation is suitable. The RMS values of the axial velocity agree well in the first 2 measurement heights. Further downstream the numerical simulation overestimates these fluctuations. 

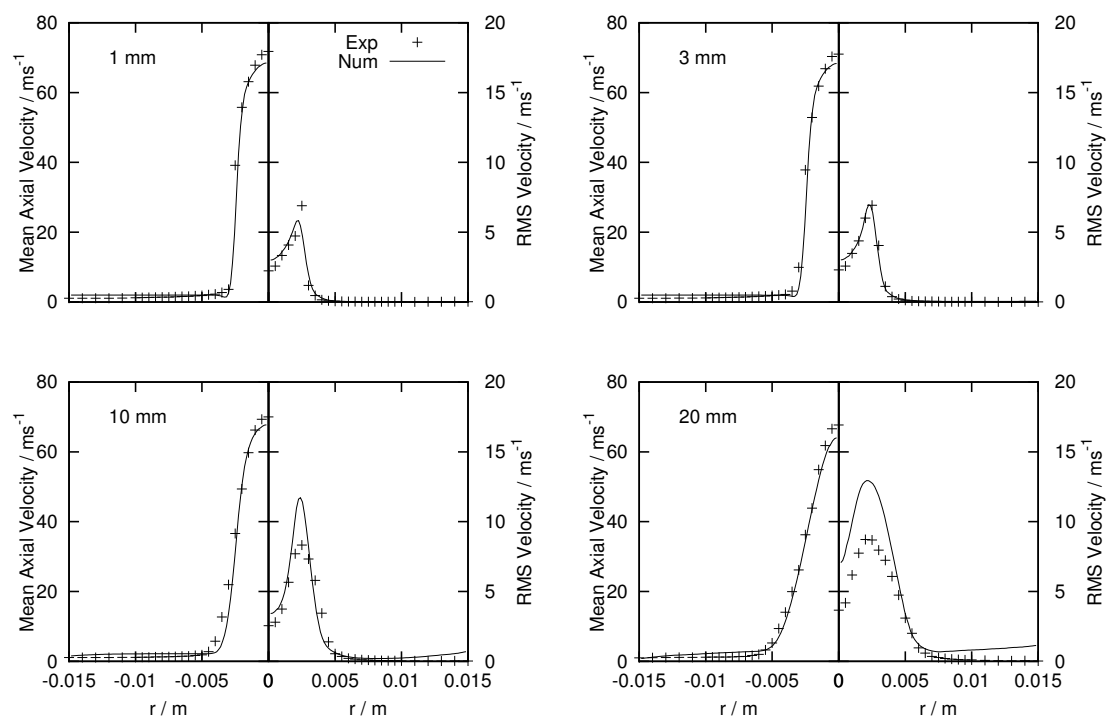

Fig. 18: Comparison of the experimental and numerical axial velocity mean and RMS of the cold jet at 4 measurement locations.

However, the structure of the flow field is captured well. Thus, the reactive simulations were performed using the same procedure. 


\section{E Differential Diffusion in the Turbulent Flame}

The comparison of a laminar flamelet solution with a scalar dissipation rate of $\chi_{s t}=1000 \mathrm{~s}^{-1}$ using a unity Lewis-number approach and a constant, but non-unity Lewis-number approach is given in Fig. 19. Differences in temperature and species mass fraction can be seen which
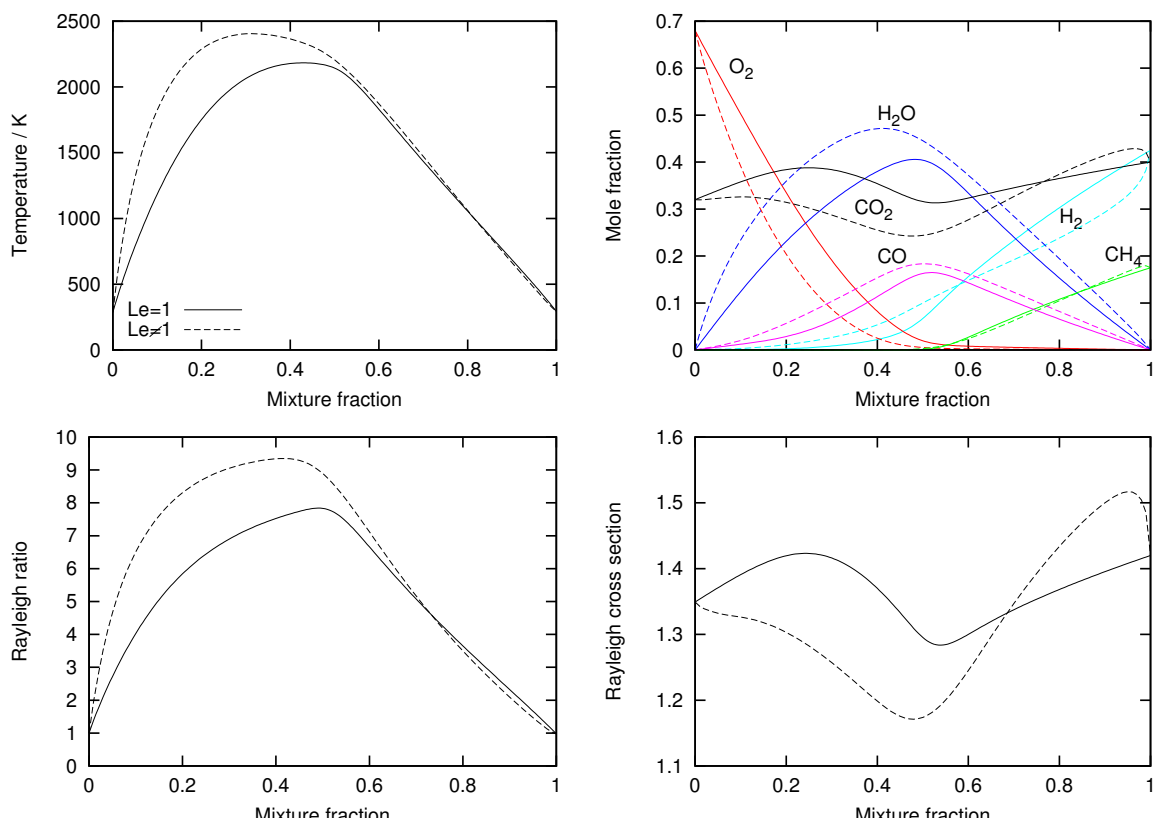

Fig. 19: Temperature, mole fraction, Rayleigh ratio and Rayleigh cross section for fuel $\left(17.5 \% \mathrm{CH}_{4}, 40 \% \mathrm{CO}_{2}, 42.5 \% \mathrm{H}_{2}\right)$ and oxidizer $\left(68 \% \mathrm{O}_{2}, 32 \% \mathrm{CO}_{2}\right)$ from a laminar flamelet solution with $\chi_{s t}=1000 s^{-1}$ using two different diffusion models.

contribute to differences in Rayleigh cross-section and signal. These differences highlight the potential for error when adopting simple assumptions to analyze the experimental data. In order to investigate the influence of differential diffusion in the turbulent flame, the Rayleigh ratio and the temperature of the laminar flamelet solutions are shown and compared in Fig. 20 for a unity Lewis-number approach and a constant, but non-unity Lewis-number approach. The Rayleigh ratio is shown in the scalar dissipation rate range between equilibrium and quenching $\left(0 \mathrm{~s}^{-1}<\chi<\chi_{q}\right)$. Very large differences in the signal can be seen due to a significant change in the species distributions. In the fuel rich region, the solutions applying different transport models approach each other. This is especially true for mixtures with $Z>0.6$. These regions can be found near the fuel nozzle. However, in the fuel lean region differential diffusion leads to significantly higher Rayleigh ratios. The non-unity Lewis-number approach leads to overall higher values in the Rayleigh ratio. Similar phenomena are found in the temperature. However, the maximum temperatures are only slightly higher for a non-unity Lewis-number approach. A significant shift of the peak temperature away from the stoichiometric mixture fraction can be seen and differences are largest on the fuel lean side.

When both transport models are applied in the turbulent simulation, a similar trend is observed in the statistics shown in Fig. 21 The maximum values near the reaction zone are overestimated by the transport model applying non-unity Lewis-numbers by at least $15 \%$. Near the fuel nozzle, both models agree well with the experimental data as expected since also the flamelet solutions approach each other. Near the nozzle and on the fuel lean side, the differential 

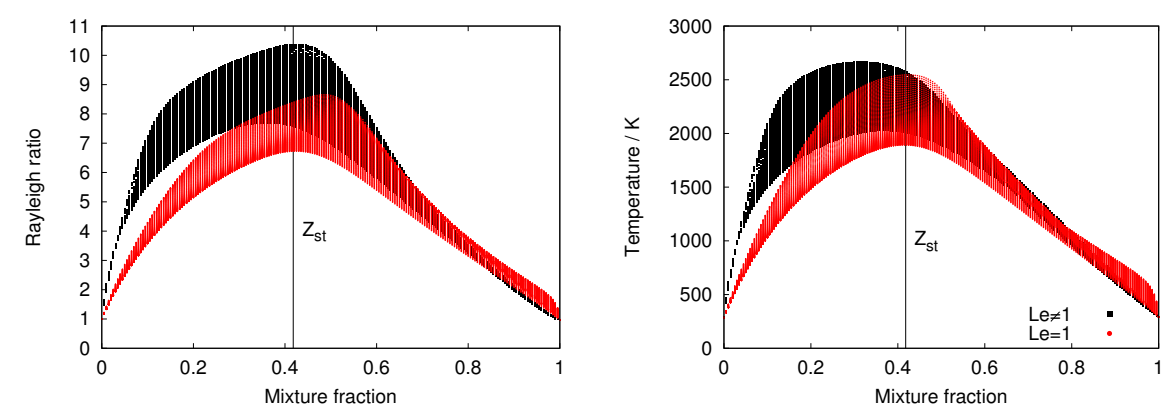

Fig. 20: Laminar flamelet solution visualisation: via the numerical Rayleigh ratio (left) and via the temperature (right) from the flamelet calculations for a unity Lewis-number approach and a constant, but non-unity Lewis-number approach.

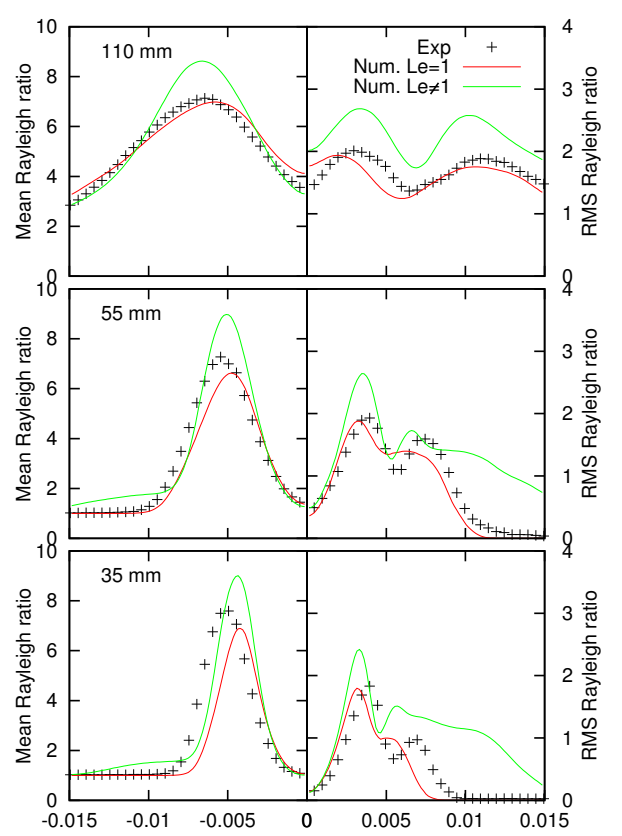

Fig. 21: Comparison of the mean (left) and RMS Rayleigh ratio (right) of the experimental data and the numerical simulation in top: $110 \mathrm{~mm}$ height, middle: $55 \mathrm{~mm}$ height and bottom: $35 \mathrm{~mm}$ height.

diffusion modeling approach overestimates the Rayleigh ratio and also shifts the gradient. Similarly, the RMS Rayleigh ratio is significantly overestimated. Much better agreement is obtained with a unity Lewis-number approach indicating that the recorded heights are less influenced by differential diffusion. 